Publications of the Astronomical Society of the Pacific, 119: 259-273, 2007 March

(C) 2007. The Astronomical Society of the Pacific. All rights reserved. Printed in U.S.A.

\title{
The Distribution of Activity on the RS CVn-Type Star SZ Piscium
}

\author{
Joel A. Eaton ${ }^{1}$ and Gregory W. Henry \\ Center of Excellence in Information Systems, Tennessee State University, Nashville, TN; eaton@donne.tsuniv.edu \\ Received 2007 January 16; accepted 2007 February 21; published 2007 March 19
}

\begin{abstract}
We use contemporaneous spectra and $B V$ light curves to derive a model of the distribution of spots and active regions on the cooler component of SZ Psc for the 1993-1994 season. For that epoch, both spots and active regions were visible at all rotational phases of this chromospherically active star. The lack of large distortions of Doppler profiles of optical lines argues for $\gtrsim 15$ spots with angular radii of $\leqslant 8^{\circ}-10^{\circ}$. Transition region emission was constant to within $\pm 12 \%$ over the orbit, nor was it eclipsed appreciably. This leads us to estimate that there are at least 20 active regions, perhaps several hundred. Chromospheric Mg II emission seems to have been proportional to the unspotted area of the star, not to the area of spots visible. We detected one strong flare in transition region lines during this program. We also present new light-curve and radial velocity solutions. These solutions find the more massive, cool star very close to its Roche lobe, and the hot star rotating more slowly than synchronously, making SZ Psc an important system for tecent he structure and evolution of binary stars. Changes in the radial velocities over 2 years of subsequent monitoring show that SZ Psc is a triple system. $\mathrm{H} \alpha$ emission seems independent of phase on the average, but it can increase markedly for periods of a few orbital cycles.
\end{abstract}

\section{INTRODUCTION}

All rapidly rotating, cool, convective stars appear to be highly spotted (e.g., Hall 1976), and this spottedness once seemed to be organized into one or two large, dark spots or spot groups (e.g., Zeilik 1992; Henry et al. 1995). However, more recent evidence suggests the spots are actually much smaller and more plentiful than those found in such classical one- or two-spot solutions. Doppler images of stars tend to find a more complicated distribution of surface brightness than two continental spots (see, e.g., Strassmeier et al. 1993b; Kürster et al. 1994; Hatzes 1995; Strassmeier 2004), and solutions of light curves, especially when combined with contemporaneous line profiles, imply much more complicated spot distributions in eclipsing systems (see Noah et al. 1987; Eaton et al. 1993; Rodonò et al. 1995). The emissions of active regions in these chromospherically active stars likewise seem to be both numerous and fairly uniformly distributed over the star (e.g., Sciortino et al. 1994; Schmitt \& Stern 1994), although there is some evidence they can occasionally be correlated with spots, as in the Sun (e.g., Baliunas \& Dupree 1982). Furthermore, random distributions of many spots with finite lifetimes, evolving under differential rotation, can explain most of the photometric variations of chromospherically active stars (Eaton et al. 1996).

We have undertaken a synoptic study of SZ Psc, a bright RS CVn binary (Hall 1976), to determine the relationship between spots and emission of the active regions in a particularly

\footnotetext{
${ }^{1}$ Guest observer with the International Ultraviolet Explorer (IUE) satellite and visiting observer at the National Solar Observatory.
}

active star. There are precious few eclipsing chromospherically active binary systems. We chose SZ Psc because it is the only one with a single active component bright enough to observe effectively with the International Ultraviolet Explorer (IUE). The system consists of a spotted, chromospherically active K1 subgiant and a much less luminous, inactive F5 IV star (Strassmeier et al. 1993a). Optical photometry of the star over its 4 day rotational cycle gives some idea of the distribution of dark starspots on the surface of the cool component. Rotational modulation of the chromospheric and transition region emission lines gives us an idea of the gross distribution of active regions on the K star. Eclipses hold at least some promise of resolving the spots and active regions. To wit, Doyle et al. (1994b) obtained IUE spectra of SZ Psc for 1990 and 1991, finding variation in Mg II strength, possibly phase dependent, and an apparent eclipse of a plage in $\mathrm{Mg}$ II. With the exception of one bright flare spectrum, their seven short-wavelength IUE spectra gave $C$ iv $\lambda 1550$ emission constant to $\pm 9 \%$.

This star is difficult to observe effectively because of an integral-day period. The most nearly complete light curves have resulted from an international campaign (Eaton et al. 1982) and from very diligent monitoring (Tunca 1984). Since then, it has been observed sporadically, notably by Lanza et al. (2001). Lanza et al. and Kang et al. (2003) have analyzed photometry for it and have given light-curve/spot solutions. While these solutions are definitely better than the previous ones based on the Russell model, they probably underestimate the radius of the K star, thus biasing the spot distributions derived. The unfortunate period also means there are relatively few measured times of minimum, so the existing period studies for SZ Psc 
TABLE 1

Optical (NSO) Spectra of SZ PsC

\begin{tabular}{|c|c|c|c|c|c|}
\hline HJD $(2,440,000+)$ & $\begin{array}{c}\mathrm{RV}_{\text {hot }} \\
\left(\mathrm{km} \mathrm{s}^{-1}\right)\end{array}$ & $\begin{array}{c}\mathrm{RV}_{\text {cool }} \\
\left(\mathrm{km} \mathrm{s}^{-1}\right)\end{array}$ & HJD $(2,440,000+)$ & $\begin{array}{c}\mathrm{RV}_{\text {hot }} \\
\left(\mathrm{km} \mathrm{s}^{-1}\right)\end{array}$ & $\begin{array}{c}\mathrm{RV}_{\text {cool }} \\
\left(\mathrm{km} \mathrm{s}^{-1}\right)\end{array}$ \\
\hline 8853.7155 & -58.0 & 66.3 & 9302.7866 & 88.7 & -37.3 \\
\hline 8853.7265 & -56.5 & 65.0 & 9302.8179 & 92.0 & -39.0 \\
\hline 8853.7397 & -54.0 & 61.7 & 9326.8146 & 109.5 & -60.2 \\
\hline 8853.7793 & -47.0 & 58.5 & 9327.6232 & 81.5 & -34.5 \\
\hline 8853.7918 & -45.0 & 58.5 & 9328.7047 & -73.5 & 78.5 \\
\hline 8853.8138 & -41.0 & 57.2 & $9328.7727 \ldots \ldots \ldots$ & -78.0 & 84.0 \\
\hline $8854.7175 \ldots \ldots \ldots$ & 92.0 & -58.0 & $9329.6566 \ldots \ldots \ldots$ & -47.5 & 59.5 \\
\hline 8854.7286 & 92.5 & -57.5 & 9329.7386 . & -34.5 & 50.5 \\
\hline 8854.8182 & 101.5 & -59.5 & 9344.6326. & -80.5 & 81.0 \\
\hline 8854.8578 & 104.0 & -63.2 & 9345.5727 & -42.0 & 52.0 \\
\hline 8854.9689 & 110.5 & -67.0 & 9355.6800 & 38.0 & -0.3 \\
\hline 8856.7547 & -75.5 & 74.2 & 9356.5856 & -85.5 & 86.5 \\
\hline 9280.8563 & -41.5 & 61.5 & 9357.5512 & -26.0 & 24.0 \\
\hline 9280.8667 & -44.0 & 60.7 & 9359.5590 & 49.3 & -7.5 \\
\hline 9280.8883 & -46.3 & 64.5 & $9360.5776 \ldots \ldots$. & -87.5 & 82.5 \\
\hline $9281.9347 \ldots \ldots$. & -61.7 & 82.5 & $9382.6485 \ldots \ldots$ & 118.0 & -67.0 \\
\hline 9282.7222 & 54.0 & -0.3 & $9382.6641 \ldots \ldots \ldots$ & 114.0 & -58.8 \\
\hline 9282.7343 & 56.0 & -0.5 & $9383.5949 \quad \ldots \ldots \ldots$ & $\ldots$ & 18.5 \\
\hline $9282.7489 \quad \ldots \ldots \ldots$ & 58.7 & -1.0 & $9384.5824 \ldots \ldots \ldots$ & -93.0 & 91.2 \\
\hline $9302.7658 \ldots \ldots \ldots$ & 85.7 & -35.0 & & & \\
\hline
\end{tabular}

(e.g., Hall \& Kreiner 1980; Tunca 1984; Kalimeris et al. 1995) are necessarily somewhat speculative because of gaps in the data. These do show, however, that the period is quite variable, as expected for an RS CVn binary (Hall 1990).

We discuss our new observations in $\S 2$ and analyze them in $\S 3$. Section 3.1 derives a new spectroscopic orbit from our radial velocities and establishes SZ Psc as a triple system; $\S 3.2$ analyzes light curves for 1978 and 1993-1994 with a spot model; § 3.3 tests this spot solution with optical line profiles; $\S 3.4$ analyzes the ultraviolet emissions of active regions; and $\S 3.5$ analyzes the variability of active regions through their $\mathrm{H} \alpha$ variations. We then summarize and discuss the results further in $\S 4$.

\section{OBSERVATIONS}

We have obtained and analyzed three types of observations: (1) high-dispersion spectra: 39 from the McMath-Pierce Solar Telescope (for 1992 and 1993-1994) plus 149 from the Tennessee State University (TSU) $2 \mathrm{~m}$ Automatic Spectroscopic Telescope (AST) (for 2004-2006), (2) $B V$ photometry from the TSU $0.4 \mathrm{~m}$ Automatic Photometric Telescope (APT), and (3) IUE spectra of prominent chromospheric emission lines for 1993 (18 low-dispersion SWP and 15 high-dispersion LWP).

\subsection{Optical Spectra}

Our 39 spectra for 1992-1993 are identified in Table 1. We also took spectra of a number of comparison stars to use as velocity standards and for simulating the composite spectra of SZ Psc. These observations were made with the stellar spectrograph of the McMath-Pierce Solar Telescope at the National Solar Observatory (NSO). They recorded the 6400-6480 $\AA$ range at a $0.16 \AA$ resolution. Some spectra were taken during a run on 1992 August 25-30, but most were obtained by staff observers as part of a monitoring program. Listed in the table are the Heliocentric Julian Date of observation (HJD) and the measured radial velocities of the two stars, hot and cool.

Figure 1 gives two sample spectra of SZ Psc showing the relative strengths of lines for the two stars, the Doppler broadening of the $\mathrm{K}$ star, and some of the structure of the lines of the K star caused by spots. Rotational broadening of the components is roughly $v \sin i=80 \mathrm{~km} \mathrm{~s}^{-1}$ for the $\mathrm{K}$ star and $v \sin i \lesssim 5 \mathrm{~km} \mathrm{~s}^{-1}$ for the $\mathrm{F}$ star. These are in contrast to values (70 and $9 \mathrm{~km} \mathrm{~s}^{-1}$, respectively) measured by Fekel (Strassmeier et al. 1993a). A. Hatzes (1995, private communication) also measured $80 \mathrm{~km} \mathrm{~s}^{-1}$.

Over the last 2 years, we have observed SZ Psc 149 times with the AST (Eaton \& Williamson 2004) at roughly the same resolution as the McMath-Pierce data. These spectra span the wavelength range 4900-7100 $\AA$ and include many photospheric lines for the two stars. Radial velocities derived from them are in Table 2, which gives the Heliocentric Julian Date of observation, measured radial velocities of the two stars, and an equivalent width of $\mathrm{H} \alpha$ of the $K$ star (in a $12.3 \AA=560 \mathrm{~km} \mathrm{~s}^{-1}$ band centered at the velocity of the $\mathrm{K}$ star).

We have obtained four new times of conjunction (cool star in front) from the radial velocity solution for the $\mathrm{F}$ star presented in the next section: HJD 2,449,284.4483 \pm 0.0027 (NSO data), HJD 2,453,420.6391 \pm 0.0032 (AST before $2,453,600$ ), HJD 2,453,702.1940 \pm 0.0012 (AST after 2,453,600), and HJD 2,440,004.755 \pm 0.012 (Popper 1988, recalculated). Combined with our time of minimum light for 1978 (HJD 2,443,823.674 \pm 0.001 from Eaton et al. 1982), 




FIG. 1.-Spectra of SZ Psc and two comparison stars. The spectra for SZ Psc were taken at phases 0.176 and 0.887 . All these spectra have been cleared of noise spikes and corrected for slopes in the continuum level. The top three curves have been translated upward by 0.5 times the continuum intensity each.

the first of these yields the ephemeris

$$
\mathrm{HJD}_{\text {obs }}=2,449,284.4483+3.965703902 \times \text { phase },
$$

which we have used in our analysis of the photometry and NSO and IUE spectra. The period is known to be variable, and recent conjunctions are early by $\sim 0.04$ days with respect to equation (1). For fitting the $\mathrm{H} \alpha$ spectra, we adopted the alternative ephemeris

$$
\mathrm{HJD}_{\text {obs }}=2,449,284.4483+3.96566356 \times \text { phase },
$$

for which the epoch is from the NSO data set and the period connects the NSO epoch to the average epoch of the two AST sets.

\subsection{Optical Photometry}

We have monitored SZ Psc since 1993 with the TSU $0.4 \mathrm{~m}$ APT (Genet et al. 1987), obtaining an extensive new light curve for 1993-1994. For this program, we collected data in the normal manner of one observation per night (three integrations for the variable, four for the comparison, HD 219018, and two for the check star, HD 218527) and let a light curve accumulate over the whole observing season. Figure 2 compares light and color curves for 1993-1994 and 1978. It contains 62 observations for 1993-1994. External precision of such nightly means is roughly $\sigma=0.004$ mag per point in both $\Delta V$ and $\Delta B$ (Henry 1995). One readily notes the striking change in brightness between the 2 years and might suspect the data are on such different photometric systems that there is a large zeropoint shift between them. Such a shift greater than $\sim 0.01 \mathrm{mag}$ is unlikely, however, since the 1978 light curve was pieced together from an international campaign (and thus is based on several independently determined transformations), and the photometric system for the APT data is maintained through nightly observations of tens of standard stars.

Lanza et al. (2001) have published photometry for the years up to 1998 that agrees reasonably well with the level of variability in our own data. The star shows the same kind of intrinsic light variation as other chromospherically active binary stars; compare Figure 3 for SZ Psc with plots for other stars, such as those in Henry et al. (1995, Figs. 2, 7, 13, and 18), Olah \& Strassmeier (2002), or Olah et al. (1997, Fig. 7). One may notice in Figure 3 an embarrassing lack of data for the recent times we were collecting spectra. This unfortunate gap was an unintended result of modifying the target list for the APT so that SZ Psc was effectively squeezed out. However, in the most recent data available, the star was never brighter than $\Delta V=-0.42$ and therefore must have been darkened by spots at all orbital phases.

\subsection{IUE Spectra}

We also observed the star with the spectrographs on the IUE satellite contemporaneously with the APT photometry and NSO spectra to measure changes in chromospheric and transition region emission lines. Exposure times were typically 90 minutes for the low-dispersion, short-wavelength (SWP) spectra and 60 minutes for the high-dispersion, long-wavelength (LWP) spectra. Kang et al. (2003) have analyzed some, although surprisingly not all, of these long-wavelength IUE spectra. Our IUE spectra are identified in Tables 3 and 4. We also list in these two tables flux in the various transition region and chromospheric lines, measured above the zero-flux level, and the flux in the 1700-1950 $\AA$ continuum (with $1800-1840 \AA$ omitted), expressed as a magnitude $\left(-2.5 \log f_{\lambda}-21.087\right)$. We have also fit Gaussian profiles to the two Mg II lines, determined velocities of the central wavelengths of these Gaussians, and calculated the FWHM intensity from the fit. These velocities generally follow the velocity of the K star, as found by Kang 
TABLE 2

OPTICAL (AST) SPECTRA OF SZ PSC

\begin{tabular}{|c|c|c|c|c|c|c|c|c|c|c|c|}
\hline HJD $(2,400,000+)$ & $\mathrm{RV}_{\text {hot }}$ & $\mathrm{RV}_{\text {cool }}$ & $\begin{array}{c}\text { EW }(\mathrm{H} \alpha) \\
(\AA)\end{array}$ & HJD $(2,400,000+)$ & $\mathrm{RV}_{\text {hot }}$ & $\mathrm{RV}_{\text {cool }}$ & $\begin{array}{c}\text { EW }(\mathrm{H} \alpha) \\
(\AA)\end{array}$ & HJD $(2,400,000+)$ & $\mathrm{RV}_{\text {hot }}$ & $\mathrm{RV}_{\text {cool }}$ & $\begin{array}{c}\mathrm{EW}(\mathrm{H} \alpha) \\
(\AA)\end{array}$ \\
\hline $53,270.7963$ & -93.77 & 77.63 & -0.1 & $53,602.8851$ & 41.83 & -5.44 & -0.1 & $53,677.5849$ & 117.16 & -51.48 & -1.2 \\
\hline $53,271.7993$ & -14.01 & 31.95 & -0.6 & $53,604.8917$ & -8.72 & 35.73 & -0.4 & $53,679.6573$ & -78.30 & 90.29 & -0.3 \\
\hline $53,272.7979$ & 110.07 & -68.78 & 0.1 & $53,607.7674$ & -81.41 & 80.05 & 0.4 & $53,680.6608$ & 61.37 & -13.19 & -1.0 \\
\hline $53,274.8379 \ldots \ldots$ & -95.46 & 75.38 & 0.3 & $53,608.7479 \ldots \ldots$ & -25.01 & 51.52 & 0.1 & $53,681.7756$ & 100.74 & -42.34 & -1.2 \\
\hline $53,279.7762 \ldots \ldots$ & -5.67 & 21.06 & -2.8 & $53,609.7249$ & 109.13 & -56.37 & -0.6 & $53,682.8033$ & -50.17 & 61.79 & -1.6 \\
\hline $53,282.8706 \ldots \ldots$ & -96.37 & 76.13 & 0.5 & $53,610.8840$ & 30.78 & 5.01 & -0.2 & $53,684.5844$ & 54.67 & -12.06 & -1.5 \\
\hline $53,287.7233 \ldots \ldots$ & -4.04 & 22.18 & -0.3 & $53,611.6409$ & -75.68 & 75.24 & 0.2 & $53,685.6553$ & 109.57 & -23.69 & -3.7 \\
\hline $53,292.6510 \ldots \ldots$ & 111.00 & -67.31 & -0.3 & $53,612.8541 \ldots \ldots$ & -4.62 & 33.88 & 0.6 & $53,686.6547$ & -34.79 & 53.76 & -2.5 \\
\hline $53,297.8082$ & -7.84 & 21.01 & $\ldots$ & $53,613.9503 \ldots \ldots$ & 118.72 & -61.81 & -0.4 & $53,687.6116$ & -76.25 & 87.14 & -0.8 \\
\hline $53,302.7013$ & -97.04 & 81.72 & 0.0 & $53,614.7609$ & 44.93 & -9.31 & -0.2 & $53,688.6269$ & 67.64 & -21.36 & -0.7 \\
\hline $53,303.7099$ & 18.35 & 6.36 & 0.2 & $53,619.8390$ & -88.40 & 82.98 & -0.3 & $53,689.6434$ & 106.88 & -45.29 & -2.5 \\
\hline $53,309.7007$ & -9.71 & 19.12 & -0.1 & $53,626.8163$ & 21.74 & 12.66 & -0.7 & $53,690.6466$ & -38.41 & 55.75 & -0.9 \\
\hline $53,313.8064 \ldots$. & -29.75 & 31.35 & -0.6 & $53,627.7136 \ldots \ldots$ & -87.07 & 84.02 & -0.3 & $53,691.5916 \ldots \ldots$ & -74.82 & 89.33 & -0.4 \\
\hline $53,319.6560 \ldots \ldots$ & 30.99 & -14.07 & 0.4 & $53,629.7117 \ldots \ldots$ & 118.11 & -58.79 & -0.7 & $53,692.6434 \ldots \ldots$ & 74.10 & -27.42 & -1.3 \\
\hline $53,328.6651 \ldots \ldots$ & 105.58 & -59.45 & -1.5 & $53,630.7274 \ldots \ldots$ & 29.22 & 5.55 & -0.3 & $53,693.6354 \ldots \ldots$ & 104.30 & -44.53 & -3.0 \\
\hline $53,329.6343 \ldots \ldots$ & -23.47 & 28.68 & -0.8 & $53,631.7327 \ldots \ldots$ & -88.47 & 86.14 & 0.0 & $53,694.6817 \ldots \ldots$ & -47.63 & 61.55 & -1.1 \\
\hline $53,339.7118 \ldots \ldots$ & 65.86 & -35.09 & -1.1 & $53,632.7280 \ldots \ldots$ & 4.62 & 25.23 & 0.7 & $53,699.6518 \ldots \ldots$ & -62.45 & 85.28 & 0.7 \\
\hline $53,350.6253 \ldots \ldots$ & -77.28 & 72.40 & 0.3 & $53,636.7531 \ldots \ldots$ & 14.16 & 15.41 & -0.9 & $53,724.6152$ & 103.65 & -46.16 & -1.3 \\
\hline $53,364.6153 \ldots \ldots$ & 84.29 & -46.68 & -1.2 & $53,637.6858 \ldots \ldots$ & 120.44 & -59.16 & -1.3 & $53,725.6025 \ldots \ldots$ & 77.47 & -23.50 & -1.0 \\
\hline $53,387.6098 \ldots \ldots$ & 100.75 & -60.42 & -1.0 & $53,638.7216 \ldots \ldots$ & 21.80 & 14.06 & -1.0 & $53,726.6022$ & -67.97 & 75.12 & -0.6 \\
\hline $53,388.6102$ & 57.02 & -21.25 & -0.8 & $53,639.6374 \ldots \ldots$ & -87.82 & 84.21 & -0.3 & $53,727.6024$ & -39.42 & 67.18 & 0.2 \\
\hline $53,483.9839 \ldots \ldots$ & 27.23 & 5.53 & -0.9 & $53,640.8875 \ldots \ldots$ & 42.02 & -4.36 & -0.7 & $53,728.6617$ & 111.32 & -52.66 & -1.0 \\
\hline $53,486.9721 \ldots \ldots$ & 115.24 & -61.38 & -0.8 & $53,641.7080 \ldots \ldots$ & 120.43 & -60.76 & -1.4 & $53,729.5964$ & 73.79 & -18.21 & -1.8 \\
\hline $53,496.9480 \ldots \ldots$ & -94.57 & 86.28 & 0.0 & $53,642.6350 \ldots \ldots$ & 30.64 & 4.58 & -0.5 & $53,730.6121 \ldots \ldots$ & -71.86 & 72.10 & -0.8 \\
\hline $53,500.9338 \ldots \ldots$ & -94.03 & 88.07 & -0.3 & $53,643.6340 \ldots \ldots$ & -88.16 & 86.99 & -0.8 & $53,731.5961 \ldots \ldots$ & -34.72 & 59.97 & -0.5 \\
\hline $53,501.9383 \ldots \ldots$ & 12.99 & 14.71 & 0.3 & $53,644.6334 \ldots \ldots$ & 6.77 & 23.76 & 0.3 & $53,732.5994$ & 108.34 & -46.31 & -1.1 \\
\hline $53,502.9337 \ldots \ldots$ & 116.53 & -60.09 & -1.1 & $53,646.7634 \ldots \ldots$ & 2.22 & 22.44 & -0.1 & $53,733.5902$ & 69.74 & -15.58 & -0.4 \\
\hline $53,503.9326 \ldots \ldots$ & 7.42 & 10.70 & -2.4 & $53,648.6303 \ldots \ldots$ & 12.02 & 19.23 & 0.4 & $53,734.5991$ & -74.56 & 80.96 & -0.3 \\
\hline $53,504.9534 \ldots \ldots$ & -91.99 & 85.86 & 0.2 & $53,649.7713 \ldots \ldots$ & 118.91 & -56.52 & -1.1 & $53,738.6122$ & -77.64 & 87.49 & -0.1 \\
\hline $53,506.9262$ & 116.45 & -58.26 & -0.3 & $53,651.8161 \ldots \ldots$ & -85.42 & 87.07 & 0.0 & $53,741.5985$ & 57.76 & -5.51 & -0.5 \\
\hline $53,509.9649 \ldots \ldots$ & 29.62 & 1.01 & 0.3 & $53,652.6690 \ldots \ldots$ & 24.16 & 13.24 & -0.3 & $53,742.6319$ & -80.41 & 87.31 & -0.8 \\
\hline $53,526.9626 \ldots \ldots$ & 109.20 & -53.69 & -1.9 & $53,653.6546 \ldots \ldots$ & 120.41 & -56.15 & -0.9 & $53,744.6314$ & 117.17 & -55.39 & -0.5 \\
\hline $53,527.9592 \ldots \ldots$ & -27.12 & 34.22 & -1.6 & $53,654.6817 \ldots \ldots$ & 3.24 & 23.44 & -0.5 & $53,745.5912$ & 52.53 & -2.17 & -0.6 \\
\hline $53,528.9003 \ldots \ldots$ & -86.73 & 86.01 & -0.1 & $53,655.6613 \ldots \ldots$ & -88.62 & 87.87 & -0.6 & $53,746.5920$ & -80.57 & 87.62 & -0.1 \\
\hline $53,529.8800$ & 43.25 & -8.71 & 0.1 & $53,657.6928 \ldots \ldots$ & 119.17 & -54.44 & -1.1 & $53,747.6175$ & -11.24 & 45.65 & -0.6 \\
\hline $53,530.9464 \ldots \ldots$ & 107.32 & -53.40 & -1.8 & $53,659.8213 \ldots \ldots$ & -80.41 & 86.63 & $\ldots$ & $53,752.5940$ & 119.55 & -55.50 & -0.6 \\
\hline $53,531.8596 \ldots \ldots$ & -17.42 & 31.65 & -2.6 & $53,660.7907 \ldots \ldots$ & 53.54 & -10.29 & 0.1 & $53,753.5942$ & 41.99 & -4.55 & -0.8 \\
\hline $53,532.8760$ & -86.18 & 90.41 & -0.5 & $53,663.6384 \ldots \ldots$ & -86.62 & 90.84 & -0.3 & $53,755.6110$ & -0.82 & 29.85 & -0.1 \\
\hline $53,533.8587 \ldots \ldots$ & 45.02 & -17.19 & 0.1 & $53,665.5986 \ldots \ldots$ & 119.90 & -57.39 & -0.6 & $53,756.6022$ & 120.69 & -57.14 & -1.1 \\
\hline $53,536.9096 \ldots \ldots$ & -81.41 & 85.53 & 0.3 & $53,666.7114 \ldots \ldots$ & -15.55 & 40.50 & -0.3 & $53,757.5954$ & 38.50 & 0.70 & $\ldots$ \\
\hline $53,548.9234 \ldots \ldots$ & -70.49 & 78.22 & 0.8 & $53,667.6491 \ldots \ldots$ & -84.28 & 89.71 & -0.3 & $53,762.5926$ & -85.36 & 93.66 & -0.2 \\
\hline $53,555.8890 \ldots \ldots$ & -52.65 & 59.61 & -0.3 & $53,668.7093 \ldots \ldots$ & 52.00 & -10.19 & -0.6 & $53,846.9858 \ldots \ldots$ & 25.59 & 13.46 & -0.4 \\
\hline $53,557.8788 \ldots \ldots$ & 79.39 & -37.09 & -0.1 & $53,669.6320 \ldots \ldots$ & 117.75 & -55.07 & -1.1 & $53,856.9711 \ldots \ldots$ & -2.61 & 31.10 & -0.4 \\
\hline $53,564.8394 \ldots \ldots$ & -67.00 & 78.13 & $\ldots$ & $53,670.6201 \ldots \ldots$ & -8.84 & 42.55 & -0.6 & $53,859.9512$ & 119.07 & -56.14 & -0.6 \\
\hline $53,565.8678 \ldots \ldots$ & 86.70 & -44.08 & -1.2 & $53,671.6548 \ldots \ldots$ & -82.94 & 90.27 & 0.2 & $53,860.9533$ & -6.02 & 30.80 & -0.7 \\
\hline $53,567.8756 \ldots \ldots$ & -61.38 & 67.32 & -1.0 & $53,672.6241$ & 44.39 & -8.11 & -0.7 & $53,861.9546$ & -85.12 & 90.29 & 0.1 \\
\hline $53,590.9266 \ldots \ldots$ & 50.83 & -9.44 & -0.9 & $53,673.6232 \ldots$ & 116.45 & -54.61 & -1.0 & $53,862.9463$ & 41.09 & -3.33 & -0.4 \\
\hline $53,594.8467 \ldots \ldots$ & 58.52 & -13.70 & -0.1 & $53,674.6146 \ldots \ldots$ & -12.12 & 38.46 & $\ldots$ & $53,863.9441$ & 118.02 & -56.56 & -0.5 \\
\hline $53,598.8033 \ldots \ldots$ & 59.00 & -14.24 & -1.6 & $53,675.6835 \ldots \ldots$ & -78.62 & 87.89 & -0.3 & $53,864.9469$ & -11.24 & 43.46 & -0.8 \\
\hline $53,599.8913 \ldots \ldots$ & -83.94 & 81.03 & -0.1 & $53,676.6193 \ldots \ldots$ & 47.91 & -9.04 & -0.2 & & & & \\
\hline
\end{tabular}

Note. - Velocities $\mathrm{RV}_{\text {hot }}$ and $\mathrm{RV}_{\text {cool }}$ are in $\mathrm{km} \mathrm{s}^{-1}$.

et al., although there are systematic deviations caused by interstellar absorptions. The FWHMs of the Mg II lines, determined from the Gaussians, are $1.97 \AA$ for $k$ and $1.77 \AA$ for $h$.

Figure 4 shows two short-wavelength $I U E$ spectra: one spectrum in which the star was flaring, and the average of the 14 other roughly constant spectra obtained outside primary eclipse.
The flare spectrum (SWP 48985) was recorded at HJD 2,449,283.475 (phase 0.755). It produced C IV emission $20 \sigma$ higher than the average of the other 17 observations, roughly the same flux as Doyle et al. (1994a) detected in a previous flare. All the emission lines in the SWP region seem to have been strengthened by the flare. The 1700-1950 A continuum 

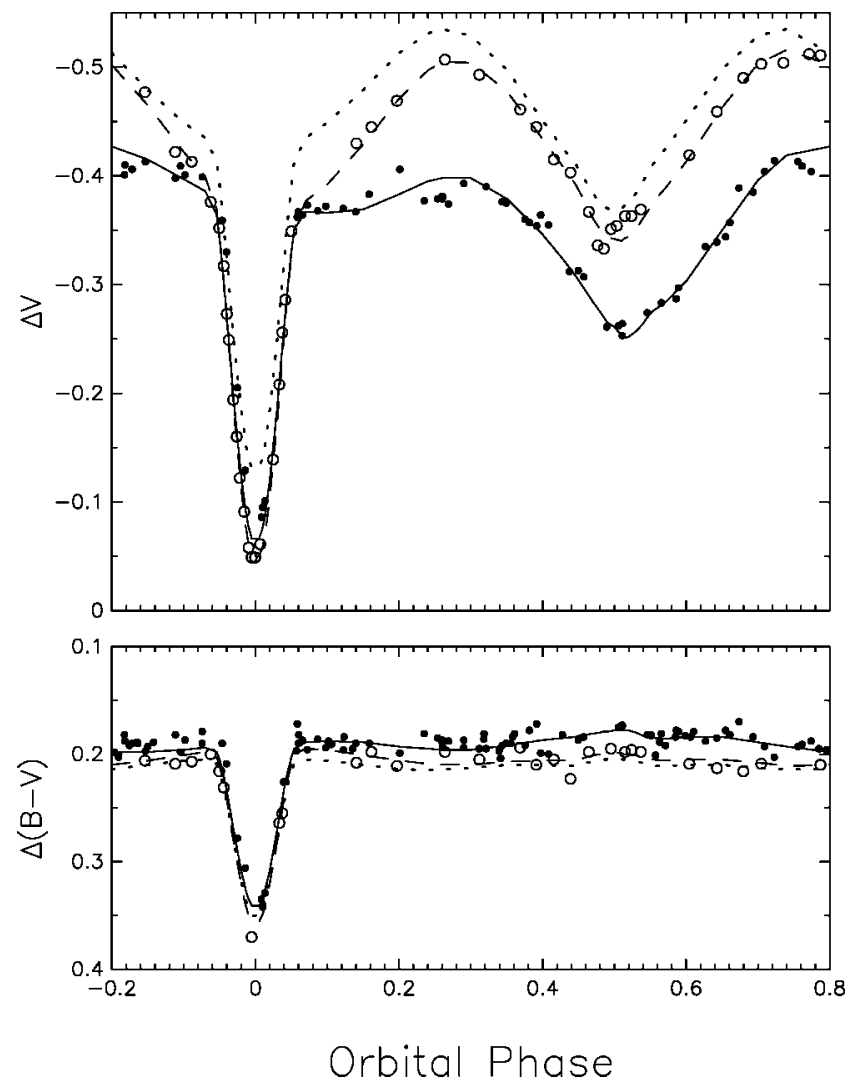

FIG. 2.-Light (top) and color (bottom) curves for SZ Psc in 1978 (from Eaton et al. 1982) and 1993-1994. Data are plotted as $\Delta V$ or $\Delta(B-V)$ vs. the orbital phase of eq. (1). Individual data for 1993-1994 are the dots; normal points for 1978 are the circles. The curves represent solutions given in Tables 6 and 7: 1993-1994 (solid), 1978 (dashed), and a completely unspotted K star (dotted). The maximum zero-point shift between the 2 years is $\sim 0.01$ mag. The only difference between the calculations is the assumed distribution of dark starspots.

band was also strengthened by $\sim 10 \%$ of the flux of the F star and decayed on a timescale of $\sim 1 \mathrm{hr}$ (see Table 4 , last column). Part of this enhancement could have been caused by unresolved emission lines (see flare spectra by Buzasi et al. [1987] and Andrews et al. [1988]).

\section{ANALYSIS OF SPECTRA AND LIGHT CURVES}

\subsection{Radial Velocities: Masses and a Third Component}

Spectra of SZ Psc are blends of spectra of a sharp-lined F5 IV star and a rather highly rotationally broadened K1 IV star. Because of the noise in our data, we decided to use crosscorrelations between the observed composite spectra of SZ Psc and spectra of slowly rotating stars of appropriate spectral type to determine the radial velocities and surface brightnesses of the two components in our NSO data. See Eaton et al. (1993) for a further discussion of the procedure. For the hotter component of SZ Psc, we took HR 8665 (= $=\xi$ Peg; F6 III-IV) as the standard; for the cooler component, we used HR 1136

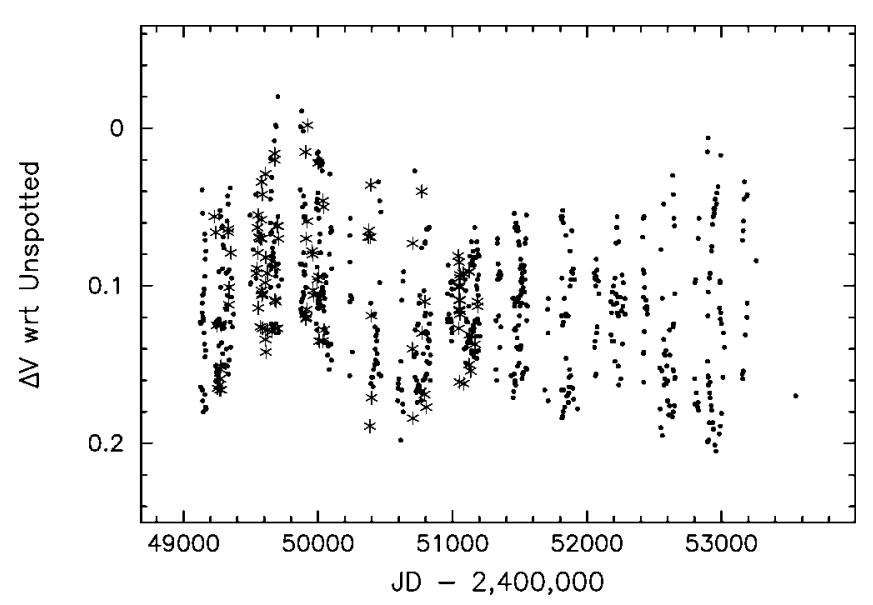

FIG. 3.-Intrinsic variation from spots using APT observations of SZ Psc outside eclipse, where $\Delta V$ refers to the phase-dependent unspotted magnitude of the system (dotted curve in Fig. 1). Dots are from the TSU APT, and asterisks are from the Catania APT (Lanza et al. 2001). Since these data include the constant light of the $\mathrm{F}$ star, the depression relative to the $\mathrm{K}$ star alone would be greater. A true believer in spot cycles will find an obvious one here.

( $=\delta$ Eri; K0 IV), rotationally broadened to $80 \mathrm{~km} \mathrm{~s}^{-1}$. We found that using rotationally broadened standards to calculate the shifts for spotted stars averages over the line profile distortions caused by spots moderately well, but some effect of the spots will always remain. We determined the radial velocities of the spectra of the two standard stars by cross-correlating them with several spectra of $\alpha$ Boo, which we assume has a constant radial velocity of $-5 \mathrm{~km} \mathrm{~s}^{-1}$. $\alpha$ Boo is a variable with a radial velocity of $-5.20 \pm 0.21 \mathrm{~km} \mathrm{~s}^{-1}$ in our AST data, transformed to the IAU system (e.g., Scarfe et al. 1990), so the velocities listed in Table 1 are probably systematically wrong by $\sim-0.20 \pm 0.21 \mathrm{~km} \mathrm{~s}^{-1}$.

For the AST spectra, we again used cross-correlation func-

TABLE 3

Long-Wavelength IUE Spectra of SZ PsC

\begin{tabular}{|c|c|c|c|c|c|}
\hline IUE Image & $\begin{array}{c}\text { Date } \\
\text { (yr/day) }\end{array}$ & HJD $-2,440,000$ & Phase & $f_{k}^{\mathrm{a}}$ & $f_{h}^{\mathrm{a}}$ \\
\hline LWP26571 ...... & $1993 / 290$ & 9278.484 & 0.496 & 384 & 325 \\
\hline LWP26572 „...... & $1993 / 291$ & 9278.595 & 0.524 & 402 & 331 \\
\hline LWP26573 ...... & $1993 / 291$ & 9278.707 & 0.552 & 421 & 327 \\
\hline LWP26574 ...... & $1993 / 291$ & 9278.817 & 0.580 & 336 & 307 \\
\hline LWP26593 ...... & $1993 / 294$ & 9282.497 & 0.508 & 443 & 338 \\
\hline LWP26594 & $1993 / 295$ & 9282.616 & 0.538 & 441 & 310 \\
\hline LWP26595 & $1993 / 295$ & 9282.732 & 0.567 & 439 & 344 \\
\hline LWP26596 ...... & $1993 / 295$ & 9282.848 & 0.596 & 394 & 334 \\
\hline LWP26601 ...... & $1993 / 295$ & 9283.420 & 0.741 & 484 & 418 \\
\hline LWP26602 ...... & $1993 / 296$ & 9283.531 & 0.769 & 539 & 483 \\
\hline LWP26603 ...... & $1993 / 296$ & 9283.642 & 0.797 & 512 & 415 \\
\hline LWP26611 ...... & $1993 / 296$ & 9284.502 & 0.013 & 490 & 386 \\
\hline LWP26612 ...... & $1993 / 297$ & 9284.618 & 0.043 & 525 & 399 \\
\hline LWP26619 ......... & $1993 / 297$ & 9285.495 & 0.264 & 555 & 451 \\
\hline LWP26620 ...... & $1993 / 298$ & 9285.606 & 0.292 & 501 & 367 \\
\hline
\end{tabular}

${ }^{\text {a }}$ Fluxes are in $10^{-14} \mathrm{ergs}^{-2} \mathrm{~s}^{-1}$. 
TABLE 4

Line Fluxes in Short-Wavelength IUE Spectra of SZ Psc

\begin{tabular}{|c|c|c|c|c|c|c|c|}
\hline IUE Image & HJD $-2,440,000$ & Phase & $f(\mathrm{O}$ I $) \lambda 1305$ & $f(\mathrm{C}$ II $) \lambda 1335$ & $f(\mathrm{C}$ IV $) \lambda 1550$ & $f(\mathrm{He}$ II $) \lambda 1640$ & $m_{\text {cont }}^{\text {a }}$ \\
\hline SWP48941 & 9278.428 & 0.482 & 25.9 & 22.3 & 54.7 & 33.9 & 12.61 \\
\hline SWP48942 ...... & 9278.540 & 0.510 & 25.3 & 21.3 & 53.1 & 23.9 & 12.63 \\
\hline SWP48944 ...... & 9278.762 & 0.566 & 17.4 & 22.6 & 42.5 & 30.4 & 12.62 \\
\hline SWP48945 ..... & 9278.857 & 0.590 & $\ldots$ & $\ldots$ & 59.1 & $\ldots$ & 12.58 \\
\hline SWP48978 & 9282.673 & 0.552 & 15.5 & 26.8 & 47.5 & 26.4 & 12.67 \\
\hline SWP48979 & 9282.790 & 0.582 & 21.8 & 12.6 & 26.4 & 22.1 & 12.68 \\
\hline SWP48985 & 9283.475 & 0.755 & 37.5 & 45.7 & 173.0 & 43.1 & 12.51 \\
\hline SWP48986 ...... & 9283.586 & 0.783 & 27.8 & 22.5 & 54.0 & 29.5 & 12.57 \\
\hline SWP48987 ..... & 9283.697 & 0.810 & 25.8 & 27.6 & 46.9 & 24.9 & 12.61 \\
\hline SWP49007 ..... & 9285.433 & 0.250 & 22.5 & 49.8 & 38.5 & 31.1 & 12.60 \\
\hline SWP49008 ..... & 9285.551 & 0.278 & 29.1 & 33.7 & 52.7 & 36.8 & 12.60 \\
\hline SWP49009 ...... & 9285.666 & 0.307 & 24.5 & 31.5 & 50.1 & 26.5 & 12.65 \\
\hline
\end{tabular}

Note. - Line fluxes are in $10^{-14} \mathrm{ergs}_{\mathrm{cm}}^{-2} \mathrm{~s}^{-1}$.

${ }^{a}$ Magnitude corresponding to flux in the 1700 to $1950 \AA$ continuum band.

tions (CCFs) to analyze the radial velocities, this time a correlation with a group of 74 prominent solar metallic lines spanning the full wavelength range of the spectra. We fit the peaks for the two components in these CCF's with Gaussians to give radial velocities of the stars. These velocities are compromised by the severe blending that occurs at many phases; however, they should be at least as precise as those from NSO. To mitigate this problem, we fit the sharp peak for the F star in the CCF first, then removed the data for it from the $\mathrm{CCF}$ before fitting the broad peak for the $\mathrm{K}$ star. The measured

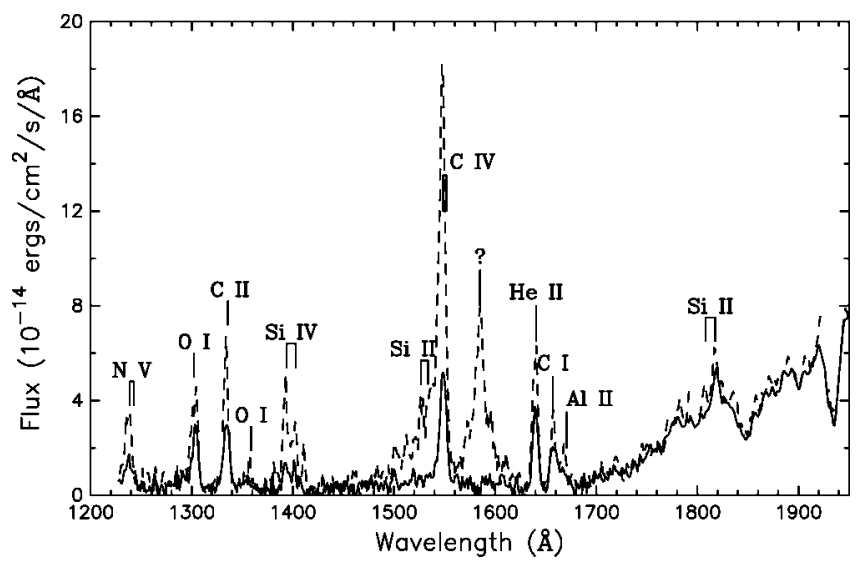

FIG. 4.- Short-wavelength IUE spectra of SZ Psc. The solid curve is an average of the 14 constant spectra taken outside primary eclipse. The dashed curve is the flare spectrum SWP 48985. The feature at $1585 \AA$ in the flare spectrum remains unidentified. It could be a radiation hit, but it is on the spectrum in a plot of the image and has the same shape as the actual identified lines. Line identifications are from Danezis et al. (1992) for the quiescent spectrum and from Doyle et al. (1989) for the flare lines. velocities in Table 2 have a $-0.35 \mathrm{~km} \mathrm{~s}^{-1}$ systematic shift with respect to the IAU velocity scale. We have corrected the systemic velocities derived from AST data ( $\gamma$ 's in Table 5) for this shift by adding 0.35 to them.

Radial velocities derived for the two components are listed in Tables 1 and 2 and are plotted in Figure 5. We fit an orbit, assumed circular, to the velocities of each star, separated into three sets: (1) NSO data, (2) AST data earlier than JD 2,453,600, and (3) AST data later than JD 2,453,600. The phasing of these orbits gave the times of conjunction quoted in $\S 2.1$ above. These individual solutions are the first three columns of Table 5. The two data sets from the AST gave systemic velocities differing by a huge $6 \mathrm{~km} \mathrm{~s}^{-1}$, and further investigation showed that the systemic velocity had increased roughly linearly over the 2 years of observation. We represented this increase with the equation

$$
\gamma=10.6+6.0[(\mathrm{HJD}-2,453,420) / 282],
$$

then subtracted this $\gamma$ (eq. [3]) from the AST data and $\gamma=$ $13.2 \mathrm{~km} \mathrm{~s}^{-1}$ from the NSO data, to give a combined data set roughly cleared of systematic errors and variations of the systemic velocity. A solution to this combined data set is given in the fourth column of Table 5. It is also plotted in Figure 5.

The amplitude of the velocity curve for the F star agrees very well with a previous study by Popper (1988). For the K star, we find a significantly smaller amplitude than Popper measured for either Ca II emission or photospheric absorption lines, implying a bigger mass ratio. We have reanalyzed Popper's data and give the results in Table 5 . We derived $K, \gamma$, and time of conjunction for the F star; for the K star, we kept $\gamma$ fixed, as had Popper, and derived $K$ for the combined measurements 
TABLE 5

Radial Velocity Solutions

\begin{tabular}{|c|c|c|c|c|c|}
\hline Quantity & $\begin{array}{c}1993 \\
\text { F Star/K Star } \\
(1)\end{array}$ & $\begin{array}{l}\text { 2004-2005 } \\
\text { F Star/K Star } \\
\text { (2) }\end{array}$ & $\begin{array}{c}\text { 2005-2006 } \\
\text { F Star/K Star } \\
\text { (3) }\end{array}$ & $\begin{array}{c}\text { Combined } \\
\text { F Star/K Star } \\
\text { (4) }\end{array}$ & $\begin{array}{c}\text { Popper (1988) } \\
\text { F Star/K Star) } \\
(5)\end{array}$ \\
\hline$K\left(\mathrm{~km} \mathrm{~s}^{-1}\right)$ & $\begin{array}{l}104.0 / 79.2 \\
\pm 0.5 / \pm 1.1\end{array}$ & $\begin{array}{l}103.3 / 74.6 \\
\pm 0.1 / \pm 1.1\end{array}$ & $\begin{array}{l}103.98 / 74.2 \\
\pm 0.02 / \pm 0.7\end{array}$ & $\begin{array}{c}104.3 / 75.7 \\
\pm 0.04 / \pm 0.5\end{array}$ & $\begin{array}{c}104.3 / 84.1 \\
\pm 1.4 / \pm 1.4\end{array}$ \\
\hline$\gamma\left(\mathrm{km} \mathrm{s}^{-1}\right)$ & $\begin{array}{c}13.2 / 13.7 \\
\pm 0.3 / \pm 0.8\end{array}$ & $\begin{array}{c}11.0 / 11.7 \\
\pm 0.4 / \pm 0.8\end{array}$ & $\begin{array}{c}16.96 / 17.4 \\
\pm 0.14 / \pm 0.5\end{array}$ & $\begin{array}{c}11.02 / 11.3 \\
\pm 0.15 / \pm 0.3\end{array}$ & $\begin{array}{c}10.3 /(10.3) \\
\pm 1.3 / \ldots\end{array}$ \\
\hline Epoch (HJD) & $2,449,284.4483$ & $2,453,420.6391$ & $2,453,702.1940$ & $2,453,420.6391$ & $2,440,004.755$ \\
\hline Number of points & $38 / 35$ & $50 / 50$ & 99/99 & $187 / 182$ & $23 / 21$ \\
\hline$\sigma\left(\mathrm{km} \mathrm{s}^{-1}\right)$. & $2.0 / 4.8$ & $2.7 / 5.3$ & $1.3 / 4.5$ & $1.6 / 4.3$ & $5.2 / 6.6$ \\
\hline$q=M_{\text {cool }} / M_{\text {hot }} \ldots$ & 1.31 & 1.38 & 1.40 & 1.38 & 1.24 \\
\hline
\end{tabular}

of $\mathrm{Ca}$ II emission and photospheric absorptions. Our new determinations of these orbital elements, based on more lines and a better way of measuring velocities of the cool star, should be more reliable than Popper's values, but in the context of this star, the range of values listed in Table 5 is a sobering reminder of just how uncertain the mass ratio must remain.

The star's changing systemic velocity means it must be a triple system, but we do not have enough phase coverage to define the wide orbit. Popper seems to have detected the effects of this orbit in systematic changes of the residuals for his fit of the F star's orbit, but these residuals are actually much better defined in our redetermination of it than in the residuals listed in his Table II. With about 4 more years of data, we ought to be able to define this orbit reliably. However, in the meantime we can place limits on it from possible phasing of the three spectroscopic data sets. The measurements now in hand suggest a velocity amplitude $K \lesssim 8 \mathrm{~km} \mathrm{~s}^{-1}$ and a period $P \gtrsim 1000$ days from the length of the rise in the AST velocities and the drop in Popper's (Fig. 6). The period is likely $\lesssim 1600$ days, to keep

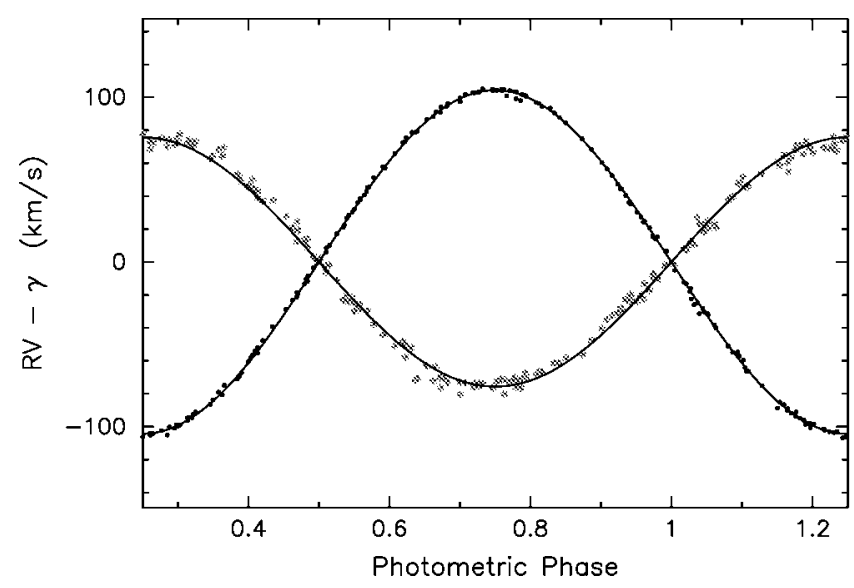

FIG. 5.-Velocity curves for the F star (dots) and K star (asterisks) derived from our combined data set, NSO and AST. The data have been corrected for the variable systemic velocity by subtracting a constant $13.2 \mathrm{~km} \mathrm{~s}^{-1}$ from the NSO data and a variable amount given by eq. (3) from the AST data. Curves represent circular orbits with $K=104.3$ and $75.7 \mathrm{~km} \mathrm{~s}^{-1}$ for the hot and cool components, respectively. the total mass of the triple system reasonable $\left(M \propto K^{3} P\right)$. Within these limits, phasing of the AST data relative to Popper's gives periods of 1143 days (12 cycles) and 1530 days (9 cycles). The NSO velocities agree reasonably well with both these periods, while ruling out possible periods between them. The rather noisy data of Jakate et al. (1976) for 1956-1957 also agree with these periods. The third body is likely to be a cool dwarf near 0.9-1.0 $M_{\odot}$, again to keep the total mass of the triple system reasonable. Such a star should contribute $\sim 5 \%$ of the light of the system near $V$ and be barely visible in a few of the strongest lines in our wavelength range, such as the $\mathrm{Na}$ I $\mathrm{D}$ lines and the $\mathrm{Mg}$ I $\mathrm{b}$ lines. We have made a composite of the (50) available AST spectra with velocities far from the center-of-mass velocity, shifted to the expected velocity of this star ( -2.8 times the velocity residual), and find weak features in the $\mathrm{D}$ lines that might well be contributed by this third body (see Fig. 7). If so, that star contributes roughly 3\%-4\% of the light of the system if the star's equivalent widths are the same

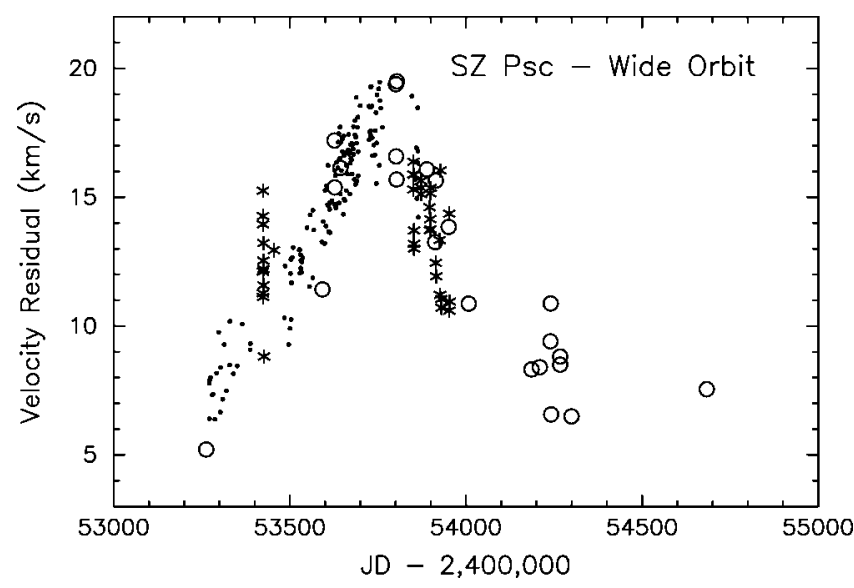

FIG. 6.-Plot of the effect of the third body on velocities of the F star. Symbols show deviations of the measured velocity from a velocity curve with $K=104 \mathrm{~km} \mathrm{~s}^{-1}$ and $\gamma=0$ for data from AST (dots), Popper (1988) (circles), and NSO (asterisks). Julian dates have been increased by 13,700 days for Popper and 4570 days $\left(P_{\text {wide }}=1143\right.$ days) for NSO. The orbital effect of a third body is clearly seen. The light-time effect in this orbit would be of the order of 0.01 days, much less than the effects of SZ Psc's intrinsically variable period. 


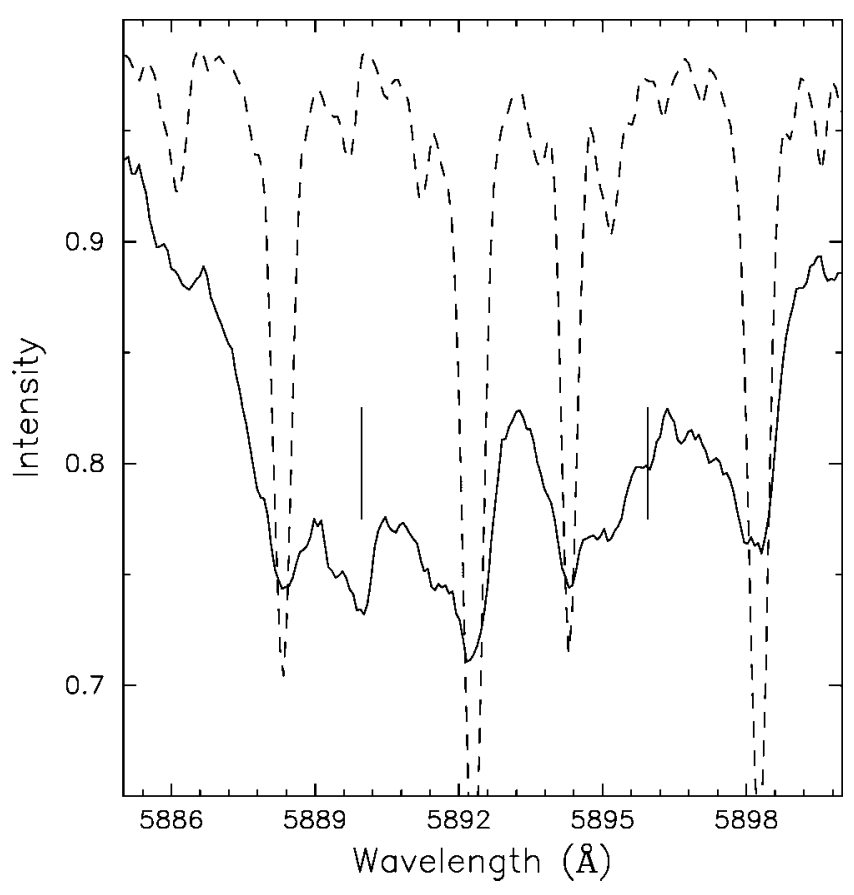

FIG. 7.-Composite of the AST spectra in which the F star is shifted far from the systemic velocity (those for which $\mathrm{RV}_{\text {hot }}>104$ or $\mathrm{RV}_{\text {hot }}<84 \mathrm{~km} \mathrm{~s}^{-1}$ ) to isolate the third star. The solid curve shows SZ Psc spectra shifted to the expected velocity of the third star. The dashed curve simulates the effect of the F star on the solid curve. (We calculated it by forming a composite in which we shifted a single F star to the orbital velocities that the F component had in those spectra we used for the solid curve.) The solid curve is shifted $-10 \mathrm{~km} \mathrm{~s}^{-1}$, which corrects for the $\gamma$-velocity of the system. With this shift, the central features of the D line lie near the rest velocities of the lines (vertical bars). The two marked features have equivalent widths of 0.029 and $0.019 \AA$, respectively.

as the Sun's. It is also worth noting that the light-time effect in this orbit would be of the order of 0.01 days, which would be difficult to detect given the variable period and uncertainties of the measured times of minimum for this star (see Kreiner et al. 2000 for an $O-C$ diagram). ${ }^{2}$

\subsection{Light Curves}

We have used the computer model of the binary described by Eaton et al. (1993) to fit the light curves and calculate line profiles. In it, the geometry of the binary is defined by the mass ratio $q=M_{\text {cool }} / M_{\text {hot }}$, the orbital inclination $i$, and the relative radii (side radii of an equipotential surface in the Roche model) $r=R / a$, where $a$ is the semimajor axis of the orbit. The surface brightness distribution in the absence of spots is determined by a limb-darkening coefficient $x_{\lambda}$, gravity-darkening exponent $g$, and bolometric albedo $A_{\text {bol }}=0.3$. The model lets us put up to 10 spots on the cool star, each bounded by

${ }^{2}$ See http://www.as.ap.krakow.pl/o-c.
TABLE 6

Properties of The SZ Psc System

\begin{tabular}{cccc}
\hline \hline Quantity & F Star & K Star & System \\
\hline$q=M_{K} / M_{F} \ldots \ldots$ & $\ldots$ & $\ldots$ & 1.31 \\
$i(\mathrm{deg}) \ldots \ldots \ldots \ldots$ & $\ldots$ & $\ldots$ & $69.75 \pm 0.5$ \\
$r=R / a \ldots \ldots \ldots \ldots$ & $0.10 \pm 0.01$ & $0.395 \pm 0.015$ & $\ldots$ \\
$a\left(R_{\odot}\right) \ldots \ldots \ldots \ldots$ & $\ldots$ & $\ldots$ & 15.2 \\
$M\left(M_{\odot}\right) \ldots \ldots \ldots \ldots$ & 1.33 & 1.74 & $\ldots$ \\
$\log \left(L / L_{\odot}\right) \ldots \ldots \ldots$ & 0.60 & $<1.09$ & $\ldots$ \\
$x_{V} \ldots \ldots \ldots \ldots \ldots \ldots$ & 0.55 & 0.71 & $\ldots$ \\
$x_{B} \ldots \ldots \ldots \ldots \ldots$ & 0.70 & 0.90 & $\ldots$ \\
$T_{\text {phot }}(\mathrm{K}) \ldots \ldots \ldots \ldots$ & 6650 & 4450 & $\ldots$ \\
$T_{\text {spot }}(\mathrm{K}) \ldots \ldots \ldots \ldots$ & $\ldots$ & 3200 & $\ldots$ \\
\hline
\end{tabular}

great circles of longitude and small circles of latitude. Parameters defining each spot are its limits in latitude $\beta$, its central longitude $\lambda$, its extent in longitude $\Delta \lambda$, and its effective temperature $T_{\text {spot }}$. We take the longitude as increasing in the direction of orbital motion from the line between the components. Thus, the phase at which a spot is closest to disk center, $\phi_{0}$, is related to central longitude $\lambda$ as

$$
\phi_{0}=0.50-\lambda / 360 \text {. }
$$

Since we do not have infrared photometry for 1993-1994, we have assumed the spots are all at $T_{\text {spot }}=3200 \mathrm{~K}, 1200 \mathrm{~K}$ cooler than the K star, as we found for 1978 (Eaton et al. 1982). Because the spots can block light at all phases, we have normalized the calculated light to roughly the average brightness the star would have outside eclipse without any spots and multiplied by a constant (added a zero point on the magnitude scale) to fit the level of the observations.

Basic properties of the binary system are given in Table 6, and the (nonunique) distributions of spots required, in Table 7. Solutions are plotted with the data in Figure 2. The geometry comes from modifying the existing light-curve solutions, the mass ratio and semimajor axis come from our radial velocity solution for 1993, the limb-darkening coefficients are estimated from model atmospheres, and the temperatures come from light-curve solutions. The previous analyses of the light curves define the geometry of the binary system somewhat ambiguously. Early solutions by Jakate et al. (1976) and Eaton \& Hall (1979) were based on limited observations. The subsequent analysis by Eaton et al. (1982) was based on a nearly complete, roughly symmetric light curve, but there were still problems with it. First, that analysis was for the Russell model, which rectifies the light outside eclipse for effects of ellipsoidal distortion of the stars. This procedure will bias to an uncertain degree the radii obtained for a system as ellipsoidally distorted as SZ Psc. Second, it took the eclipses to be complete, while new NSO and IUE spectra in the apparently total eclipse show that $\sim 30 \%$ of the light of the hot star is visible at conjunction (see Table 4, last column, and Fig. 9). Third, that analysis did not take spots sufficiently into account, since it rectified the 
TABLE 7

Multispot Solutions

\begin{tabular}{|c|c|c|c|c|c|c|}
\hline \multirow[b]{2}{*}{ SPOT NUMBER } & \multicolumn{3}{|c|}{$1977-1978$} & \multicolumn{3}{|c|}{ 1993-1994 } \\
\hline & $\beta$ & $\lambda$ & $\Delta \lambda$ & $\beta$ & $\lambda$ & $\Delta \lambda$ \\
\hline $1 \ldots$ & -9.2 to +9.2 & 90.0 & 18.0 & -21.1 to +26.1 & 79.2 & 28.8 \\
\hline $2 \ldots \ldots \ldots \ldots$ & -13.9 to +13.9 & 169.2 & 21.6 & -9.2 to +9.2 & 111.6 & 14.4 \\
\hline $3 \ldots$. & -9.2 to +9.2 & 331.2 & 21.6 & +13.9 to +34.1 & 158.4 & 21.6 \\
\hline $4 \ldots$ & $\ldots$ & $\ldots$ & $\ldots$ & -4.6 to +9.2 & 230.4 & 14.4 \\
\hline $5 \ldots$ & $\ldots$ & $\ldots$ & $\ldots$ & +9.2 to +28.7 & 273.6 & 25.2 \\
\hline $6 \ldots$ & $\ldots$ & $\ldots$ & $\ldots$ & -4.6 to +4.6 & 298.8 & 14.4 \\
\hline $7 \ldots$ & $\ldots$ & $\ldots$ & $\ldots$ & -4.6 to +13.9 & 316.8 & 21.6 \\
\hline $8 \quad \ldots \ldots \ldots \ldots$ & $\ldots$ & $\ldots$ & $\ldots$ & -9.2 to +21.1 & 345.6 & 21.6 \\
\hline
\end{tabular}

NoTES. - Photometric zero points for both years were $\Delta V_{0}=-0.490$ and $\Delta B_{0}=-0.280$. These values correspond to a combination of two unspotted spherical stars with the temperatures and relative radii in Table 5. The corresponding observed magnitude differences at maximum light are $\Delta V=-0.535$ and $\Delta B=-0.321$.

light only for those asymmetries about phase 0.0 caused by tides and reflection effect. It now appears there were spots visible near phases 0.0 and 0.25 in 1978 (compare the $V$-band curves in Fig. 2). Later solutions by Lanza et al. (2001) and Kang et al. (2003) are better, being based on a geometrically more realistic model, but they are biased by assumptions about the level of spottedness, particularly Kang et al., and they do not give $v \sin i$ as large as observed $\left(v \sin i \lesssim 61 \mathrm{~km} \mathrm{~s}^{-1}\right.$ with any mass ratio in Table 5).

The old solution for 1978 showed that the larger, cooler, more massive star fills a large fraction of its Roche lobe, that the hotter, unspotted star is near the main sequence, that the hotter star is probably somewhat hotter than implied by its F8 IV spectral type, and that the cooler star is cooler than expected for K1 IV. Trying to fit our high-dispersion line profiles in the NSO spectra simultaneously with the 1993-1994 light curve requires the $\mathrm{K}$ star to be very close to its Roche lobe (Fig. 8 compares some observed and calculated profiles). It is likely to be somewhat smaller than the Roche lobe, since a more massive contact component is presumably dynamically unstable to mass loss to its companion, but the observed rotational broadening requires the $\mathrm{K}$ star to be roughly as big as we have made it in Table 6 . The radius of the $F$ star would be determined by its rotational broadening if it were rotating synchronously $\left(v \sin i \sim 19 \mathrm{~km} \mathrm{~s}^{-1}\right)$. However, the $\mathrm{F}$ star's lines are not resolved in our spectra $\left(v \sin i \lesssim 5 \mathrm{~km} \mathrm{~s}^{-1}\right)$, and the star therefore cannot be rotating synchronously. If it were, its radius would have to be much too small for its mass or color. The relative radius and temperature of the hotter star were chosen to fit the color variation in eclipse. The inclination follows from the central depth of primary eclipse measured in IUE spectra. Our new value is significantly smaller than previous ones because of our larger relative radius for the $\mathrm{K}$ star, and from the requirement that the eclipse be partial.

The remarkable property of our light curves is the nature and magnitude of change between 1978 and 1993-1994. The former light curve requires relatively few dark starspots-only three in our solution. The huge, complicated depression observed in 1993-1994, however, requires a complicated distribution of spots-eight in our photometric solution. While this distribution is not unique, it does represent a possible collection of spots that will give the observed light loss with roughly the minimum number of spots, and the actual spots must give a similar distribution of spot area with stellar longitude.

\subsection{Photospheric Line Profiles}

Starspots will distort Doppler profiles of optical absorption lines, the effect that spawned the field of Doppler imaging. Our spectra for 1993-1994 are too noisy for forming true Doppler images, but they do give us a powerful test of distributions of spots derived from light-curve solutions, as well as a means for assessing just how large the spots are permitted to be without violating spectroscopic constraints. To perform this test, as we did for RS CVn (Eaton et al. 1993), we have calculated cross-correlation functions between the unbroadened spectrum of a single star ( $\delta$ Eri) and observed spectra of SZ Psc. These CCF's average over all the available lines of both components of the system, thereby reducing noise of the profiles but discarding any temperature dependence that may be present in different lines. We then use the model derived from the light curve to compute theoretical spectra, calculate their cross-correlation functions, and compare these calculated CCF's with those observed at the same orbital phase. See Figure 8 for some examples. Note that these spectra, for 6400-6480 A, do not include any chromospherically sensitive lines, such as $\mathrm{H} \alpha$, and should reflect conditions in the photosphere.

As with RS CVn, we have found a solution to the light curves that fits data for 2 separate years with the same geometry and spot temperatures, but with different numbers of dark spots. The distribution of spots for 1993-1994, however, requires at least eight spots in our solution, including four relatively big ones (Nos. 1, 3, 5, and 8 in Table 7, with effective angular radii of $20.6^{\circ}, 11.2^{\circ}, 12.2^{\circ}$, and $14.3^{\circ}$, respectively). Spots with 


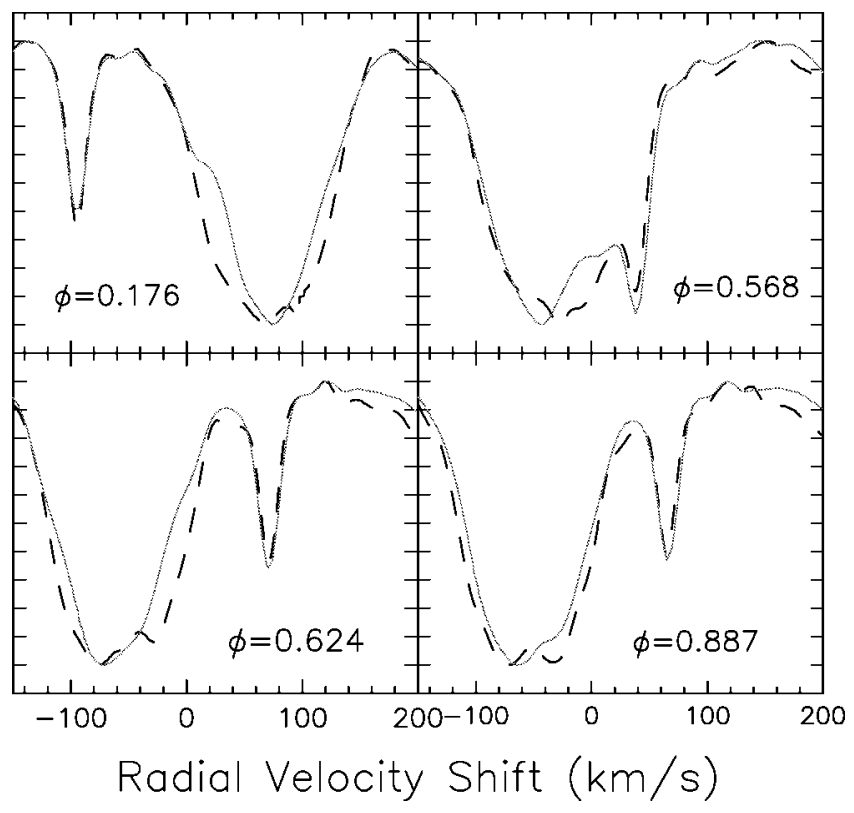

Fig. 8.-Failure of the spot model to fit observed line profiles: observed (dashed curve) and calculated (solid curve) cross-correlation functions for four phases illustrate the undetected effect of large starspots on line profiles. At phase 0.176 (top left), we see the left side of the calculated profile removed by spot No. 1 , while the right side is distorted by spot No. 3 . At phase 0.624 (bottom left), the right side of the profile is distorted by spot No. 8, while at phase 0.887 (bottom right), the right side is distorted by spot No. 5 .

such large radii are not allowed by distortions of the optical line profiles, as one can see in Figure 8. That our nonunique distribution of spots does not fit the line profiles is not particularly surprising. Yet the magnitude of the calculated distortions seen teaches an important lesson about the spots on this star, inasmuch as profile distortions in this star are hardly ever as big as those calculated for the moderately large spots in our solution. In other words, while our big spots might be moved around somewhat to decrease the deviations from observed profiles at particular phases, they would still distort other profiles too much. Instead, these big spots must break up into smaller spots roughly $8^{\circ}-10^{\circ}$ in radius to be hidden in the line profiles. The light-curve depression in 1993-1994 would require at least 15 such spots.

An alternative way to get a general dimming of the light of a star, as well as to produce the kind of square Doppler profiles seen in Figure 1, is the sort of "polar spot" found in many Doppler images of chromospherically active stars. This kind of feature must of course be augmented with enough spots at lower latitude to give the phase dependence of any light variation, but these other spots are not necessarily very big. Such polar spots will depress the overall brightness of the spotted component, as well as that of the whole binary. In SZ Psc, a $30^{\circ}$ (radius) polar spot gives $\Delta V \approx 0.05$; a $40^{\circ}$ spot, $\Delta V \approx$ 0.12 . The light curve for 1978 would not allow polar spots bigger than $\sim 15^{\circ}$, unless the unspotted brightness of the cool component is larger than we have assumed. Of course, it is entirely possible that we have underestimated the unspotted brightness and that there could be polar spots or many, many undetected tiny spots all over the star.

A symmetrical polar spot has essentially no effect on the ellipsoidal light variation of the system. This means that the suppressed ellipsoidal variation in recent years requires either discrete spots at lower latitudes visible preferentially at the quadratures, or extensions of the polar spots at those phases. The lack of large distortions in the wings of profiles in Figure 8 shows that we do not have spots preferentially near the equator, so they might be confined to high latitudes to get the overall dimming and square profiles seen. We have investigated this possibility by replacing spot Nos. 1 and 2 with two spots at high latitude (one in each hemisphere), but the line profiles are still too distorted in the wings at phase 0.17 . Breaking up the resulting spot in the more visible hemisphere further while spreading its two components in longitude helps, but doing so still gives larger line profile distortions than we observed. A further amoeba-like division, with spreading in both longitude and latitude to reduce the effect on the line profiles, is thus required, but that is beyond the scope of our techniques. So we are once again left with the conclusion that there cannot be just a few huge spots on these chromospherically active stars.

\subsection{Ultraviolet Emission Lines}

Doyle et al. (1994b) found their Mg II lines had the same velocity as the $\mathrm{K}$ star, and our $\mathrm{Mg}$ II lines also clearly follow the K star, as velocities of Gaussians fitted to the profiles and directly calculated profiles show (see also Fig. 2 of Kang et al. 2003). Figure 9 gives examples of such line profiles at four phases. We calculated the simulated line profiles by assuming that emission is evenly spread over the K star. In this approximation, most of the line broadening is intrinsic, not rotational Doppler broadening. Rotation would play a bigger role if the emission is limb brightened or if it is confined to equatorial active regions. These simulations show clearly that the emission arises on the K star-hardly unexpected in light of Popper's (1988) use of Ca II H and $\mathrm{K}$ emission to measure the velocity of that component. The calculated profiles are somewhat narrower than observed profiles in several cases. This could result either from using too narrow a local profile or from having limb-brightened equatorial sources visible at the phases in question. The IUE spectra of this star are too noisy to decide if either condition applies. In addition, the alternative profiles we calculated by assuming that the active regions coincide with the starspots of Table 7 were Doppler-shifted from the observed lines in typical cases. Furthermore, if $\mathrm{Mg}$ II emission is directly proportional to spot area, the emission would vary by a factor of 3 over the orbit versus the $\sim 10 \%$ observed. These results may indicate that the active regions do not coincide with the spots, but the actual distributions of spots must be more com- 


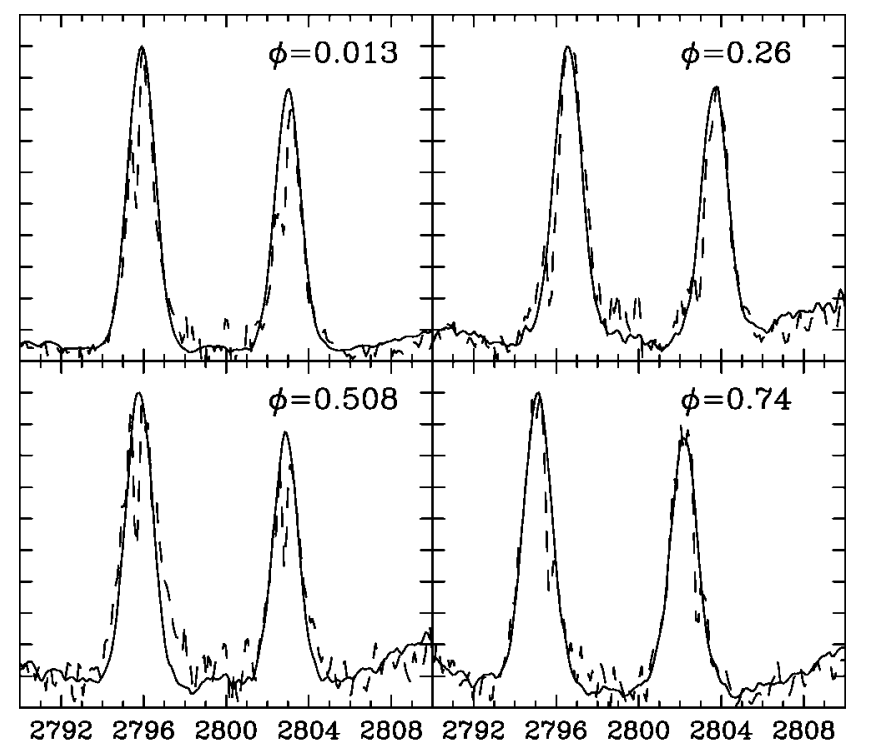

Wavelength $(\AA)$

FIG. 9.-Mg II lines at four orbital phases. Observed line profiles are shown as dashed curves, calculated profiles as solid curves. The sharp dips in the observed spectra of SZ Psc are interstellar lines, which we have not attempted to simulate. All the Mg II emission comes from the K giant. Most of the line broadening in the calculations is intrinsic, not rotational, despite the large $v \sin i \sim 80 \mathrm{~km} \mathrm{~s}^{-1}$ of the $\mathrm{K}$ star. We have simulated the intrinsic spectrum of the $\mathrm{K}$ star with an archival spectrum of $\sigma \mathrm{Gem}$, which has a relatively low $v \sin i$, and the spectrum of the $\mathrm{F}$ star with an archival spectrum of $\alpha \mathrm{CMi}$. Even so, the calculated emission-line profiles seem somewhat too narrow for the observations. Radial velocities, on the other hand, fit quite well.

plicated, possibly more uniform, so determining an association must wait for much better data.

Figure 10 shows the variation of chromospheric Mg II flux and transition region $\mathrm{C}$ IV flux with orbital phase. $\mathrm{Mg}$ II strength is roughly proportional to the brightness of the $\mathrm{K}$ star (solid lines in the figure), which means it is weakest when the spots are most prominent. This is the same as Doyle et al. (1994b) found for their four observations in 1991. C IV, which has roughly the same phase dependence as all the lines in Table 4 (but much lower relative uncertainties), is constant to within $\sigma= \pm 12 \%$.

Although SZ Psc is relatively bright, it was still a faint target for $I U E$, and our spectra were rather noisy, as were those in previous studies. For this reason, our $\mathrm{Mg}$ II line profiles are too noisy to detect eclipses of active regions reliably. We had hoped to observe such eclipses of active regions, since $4.5 \%$ of the $\mathrm{K}$ star is covered at central eclipse. Spots are eclipsed in both SZ Psc and RS CVn, but there is no evidence for eclipse of large active regions in our IUE spectra. This means that either there are so many active regions that we cannot see the dimming in noisy $I U E$ data, or there are few active regions and none was eclipsed. Doyle et al. (1994b) found a drop in

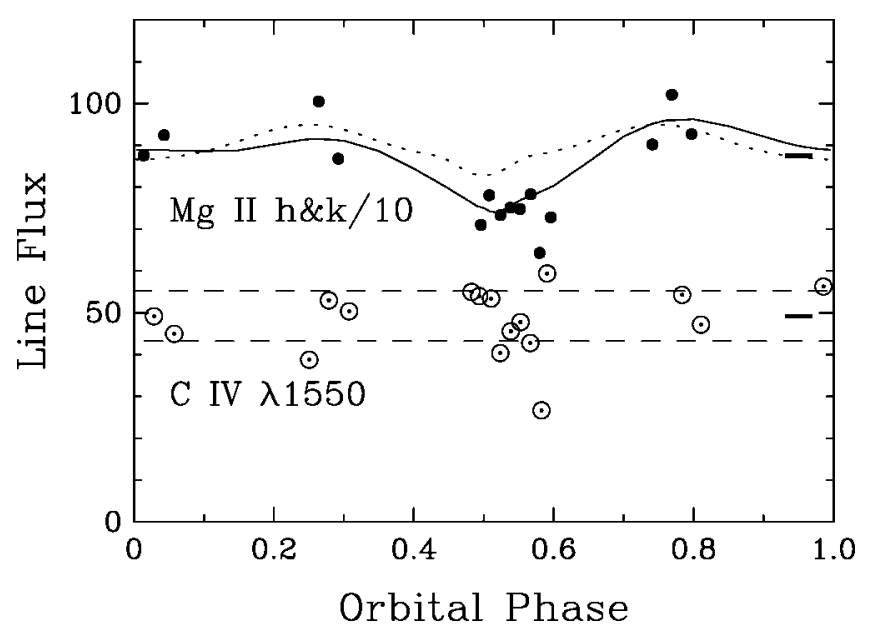

FIG. 10.-Phase dependence of chromospheric and transition region lines. Units are $10^{-14} \mathrm{ergs} \mathrm{cm}^{-2} \mathrm{~s}^{-1}$, with the total flux in $\mathrm{Mg}$ II $h$ and $k$ divided by 10. We have not attempted to correct $\mathrm{Mg}$ II for the noticeable interstellar absorption. The measured phase variation of $\mathrm{Mg}$ II is more nearly proportional to the unspotted brightness of the K star (solid curve) than to the total projected surface area of the K star (dotted curve) or to the spot area. Dashed lines for $\mathrm{C}$ IV are $\pm 1 \sigma$ limits. Heavy bars to the right of the figure give the levels of ambient emission found by Doyle et al. (1994b) for their spectra of 1990 and 1991, although we have increased their $\mathrm{Mg}$ II flux by $17 \%$ to put it on our flux scale.

Mg II flux around phase 0.5 that they took as a possible eclipse, but if about half the Mg II emission comes from an area small enough to be eclipsed, there should be much stronger, and different, phase dependence than was observed in 1991.

The $10 \%$ fluctuations of C IV for all the nonflare data (seven spectra for 1990-1991 by Doyle et al., and our 17 spectra for 1993) give a way of estimating the number of active regions. We may assume that all this variation comes from active regions rotating into and out of our view. If so, each active region will contribute $\sim 10 \%$ of the emission for a hemisphere. Therefore, there will be at least 20 active regions spread over the whole star. Phase variations may place further restrictions on the number of active regions. If such regions are randomly distributed over the star, and if all have the same luminosity, the phase variation of line flux will be, on average,

$$
\sigma_{f} /\langle f\rangle=1 / \sqrt{(\mathrm{n} / 2)}
$$

where $\sigma_{f}$ is the average of the standard deviations of the flux in a number of years, and $n$ is the number of active regions. The $\sigma_{f} /\langle f\rangle \sim 0.08$ for $1990,0.10$ for 1991 , and 0.03 for 1993 for an average of 0.07 , whence $n \sim 400$.

\subsection{The $\mathbf{H} \alpha$ Profile}

Huenemoerder \& Ramsey (1984) discussed the phase dependence of $\mathrm{H} \alpha$ using 13 spectra with $\sim 2 \AA$ resolution. A K1 IV star would normally have $\mathrm{H} \alpha$ in absorption, with an equiv- 


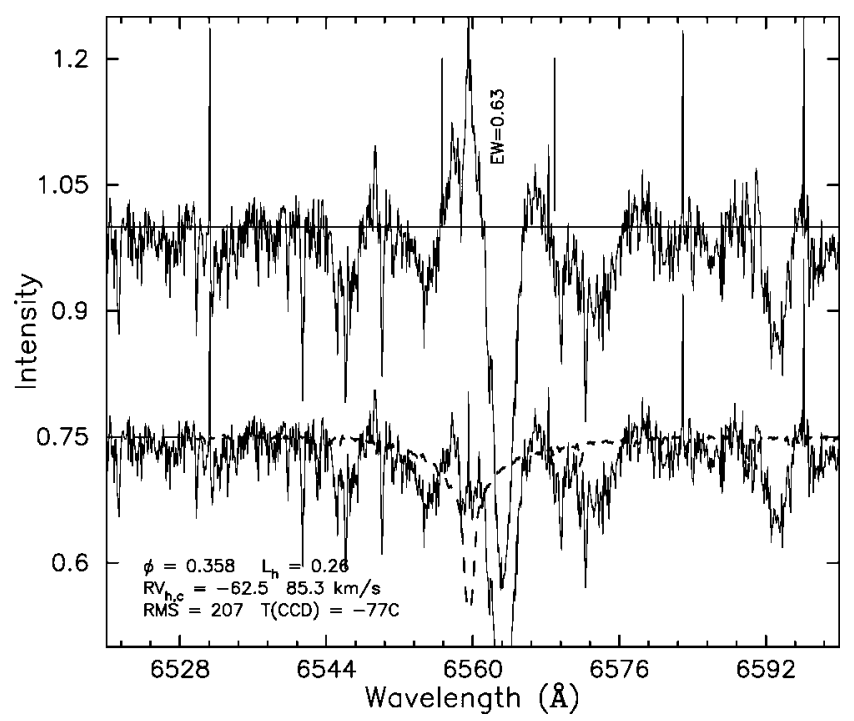

FIG. 11.-Measurement of the equivalent width of $\mathrm{H} \alpha$ in SZ Psc. This is the spectrum for JD 2,453,699. The bottom plot of the spectrum shows the placement of the continuum and the spectrum of the F star to be subtracted from the observed spectrum. The top curve is the spectrum of the $\mathrm{K}$ star alone (cleared of light of the F star). The two vertical lines show the passband we used to measure equivalent width.

alent width near $1.0 \AA$ (Eaton 1995). In SZ Psc, the strong absorption feature of the K star is usually "filled in" with emission, whatever that means. We now have 149 spectra in which we can measure $\mathrm{H} \alpha$ equivalent widths and roughly define the line profiles to look for phase dependence of the emission. To measure these, we plotted the observed spectrum, corrected the continuum level for errors caused by our pipeline reduction, subtracted the spectrum of an F star shifted to the measured velocity and scaled to the expected intensity of the hot com-

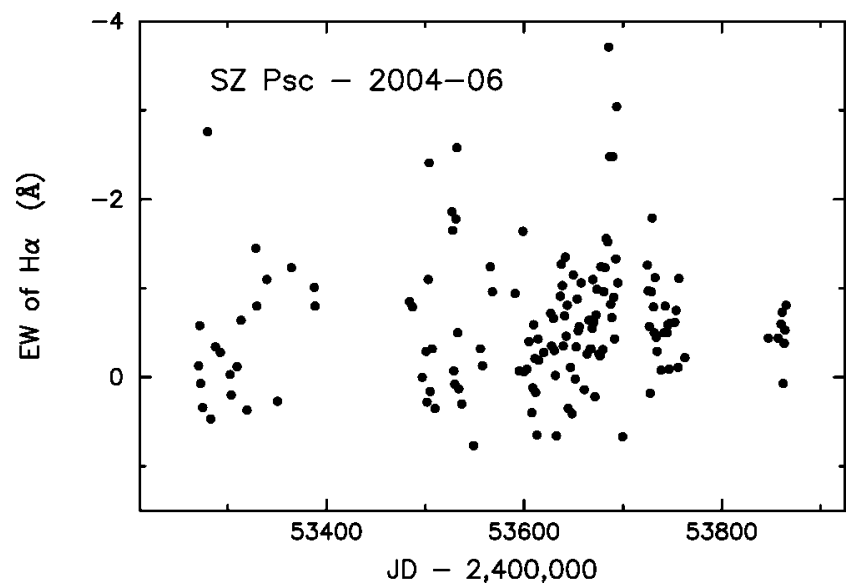

ponent ( $\gamma$ Ser; F6 V), shifted the spectrum of SZ Psc to the velocity of the cool component, and measured the equivalent width in a $12.3 \AA$ band centered on the velocity of the cool component. In this scheme, absorption equivalent widths are positive; emission, negative. The calculated red light curve used for subtracting the spectrum of the F star gave that star about $28 \%$ of the light in the continuum, on average, outside eclipse; less in primary eclipse. Given the number of steps in the process, likely errors are of the order of $0.3-0.4 \AA$ A. Figure 11 shows an example of this procedure. The main points to note are the huge width of the emission line in this star and the strong central reversal in this particular observation. The band large enough to include all the emission is about $560 \mathrm{~km} \mathrm{~s}^{-1}$ wide, 3.5 times the full rotational Doppler spread of the K star. The central absorption component is $\sim 190 \mathrm{~km} \mathrm{~s}^{-1}$ wide, roughly what one might expect for an intrinsically strong rotationally broadened line. The measured equivalent widths from Table 2 are plotted in Figure 12. In the aggregate, there was little phase dependence over the last 2 years, but this is not surprising, because spots were probably always visible (see $\S 2.2$ ). However, $\mathrm{H} \alpha$ was highly variable on timescales of several orbits in this data set. As an example of such variability, the emission was much stronger than normal near phase 0.8 on three consecutive orbits near JD 2,453,690, although it was no stronger than average on the orbits immediately before and after. This enhancement is shown in Figure 12 by the circled dots in the right-hand panel. There was no obvious emission in He I $\lambda 5876$ in these spectra or in any others we have.

Normally, $\mathrm{H} \alpha$ in the cool stars is formed mostly in the upper chromosphere, where temperatures and electron densities are high enough to excite the second level of hydrogen. At lower chromospheric densities, the source function is controlled by a balance between photoionization by dilute photospheric radiation on the one hand and recombination on the other (Jef-

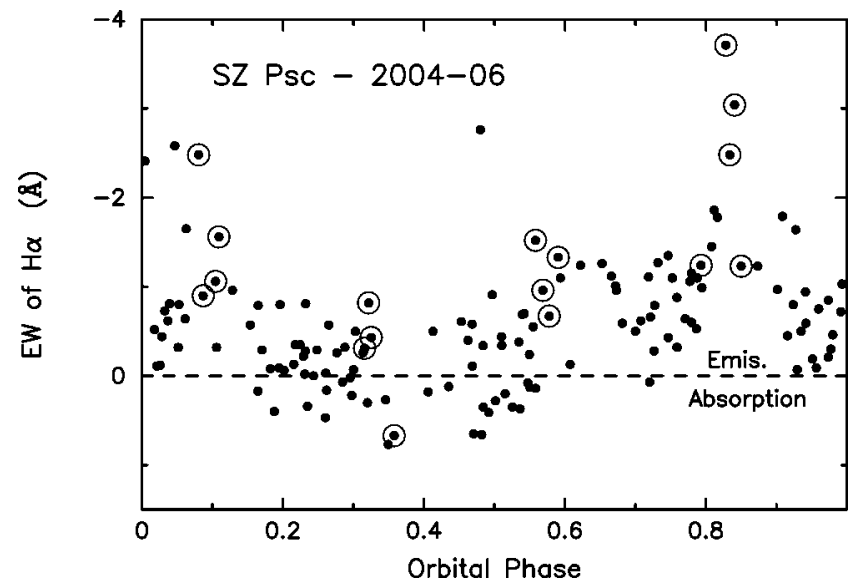

FIG. 12.-Behavior of $\mathrm{H} \alpha$ in SZ Psc. Left: Plot shows that the line was usually in emission, with a few episodes of much higher emission. Right: Plot shows a hint of phase dependence, with the emission somewhat stronger in the second half of the orbit. Circled points give the measurements near the "flare" around JD 2,453,690 and cover the cycles before and after this event. The feature near phase 0.8 appeared in three successive cycles. 
feries \& Thomas 1959; Johnson \& Klinglesmith 1965; Cuny 1965). In these circumstances, the upper levels of $H$ are not populated enough to give Balmer emission. However, as the chromosphere becomes denser and thicker, Ly $\alpha$ becomes stronger, even thermalized, in a larger amount of gas, and the optical depth of $\mathrm{H} \alpha$ increases. This initially leads to stronger absorption in $\mathrm{H} \alpha$. Eventually, with increasing temperature and electron density, collisions begin to dominate the $\mathrm{H} \alpha$ source function, and the line goes into emission (see Cram \& Mullan [1979] for an excellent discussion of all these processes, and Baliunas et al. [1979] for some further insight about such chromospheric structure). In this context, there are at least two ways of interpreting the filling in of spectral lines. First, the emission could simply result from global changes in the physical conditions in the chromosphere, so that the normal absorption line is everywhere replaced by an emission line. Alternatively, the chromosphere in general could be forming the usual $1 \AA$ absorption line, with the emission coming from small, isolated regions of much denser, probably hotter, gas. The profiles seem to favor the first of these mechanisms. The typical profile is a weak, broad emission line, as in Figure 11, but with a much weaker central absorption than in Figure 11. That the central absorption is weak means the broad emission is not simply formed in a few isolated clumps; if it were, the absorption component would be the normal absorption for a K star. Rather, the conditions over the whole disk must be altered so that the usual absorption is not formed in the first place, but is replaced with a self-reversed emission. On the other hand, the variability of $\mathrm{H} \alpha$ emission might seem to be too great to be coming from a truly global phenomenon. However, at times of greatly enhanced emission, the absorption component disappears, again meaning the emitting gas must cover most of the visible disk, or that the emission-line profile is strongly constrained to just fill in the normal absorption profile of the ambient disk.

The phase dependence of chromospheric emission in the chromospherically active stars has long been a difficult and controversial topic. One would imagine the emission would be associated with starspots, inasmuch as solar active regions are associated with spots, and that emission should be strongest when the star is faintest. However, evidence for such an association is weak in the better observed cases and usually rests on scanty data. The best evidence for it is probably the observations of $\mathrm{Ca}$ II $\mathrm{H}$ and $\mathrm{K}$ in $\lambda$ And by Baliunas \& Dupree (1982). Weiler (1978a) measured equivalent widths of $\mathrm{H} \alpha$ and $\mathrm{Ca}$ II in six RS CVn binaries, finding emission stronger at photometric-wave minimum in three systems. Weiler (1978b) then used satellite observations for two binaries to argue that Ly $\alpha$ and Mg II emissions were phase dependent. Bopp \& Talcott (1978) found a correlation of $\mathrm{H} \alpha$ emission with photometric dimming in UX Ari, but generally $\mathrm{H} \alpha$ varies erratically or is roughly constant in these stars (Bopp \& Talcott 1978, 1980).

Complicating the picture of chromospheric emission in SZ Psc is the suggestion of Bopp (1981) that the $\mathrm{H} \alpha$ in outburst is formed in a transient disk around the $\mathrm{K}$ star. There were a couple of reasons for his proposal. First, the emission line, at least in outburst, is much broader than in other chromospherically active stars, although Hatzes (1995) detected an unexplained broad $\mathrm{H} \alpha$ component in DM UMa. This observation argues that its profile does not reflect the intrinsic broadening of chromospheric gas or the rotational broadening of the surface of the star. Second, since the K giant in SZ Psc fills most of its Roche lobe, Bopp thought it might suffer episodes of mass transfer, which might somehow form a disk around the K star, perhaps through the agency of those shady magnetic fields. Jakate et al. (1976) had also advanced mass transfer as the source of a period change. Recent mass transfer, however, seems unlikely because of the slow rotation of the F star, the gainer, which normally would collect plenty of rotational angular momentum from the accreting mass (see analyses such as Van Hamme \& Wilson [1993] for Algol binaries). Furthermore, plots such as Figure 11 show that the emission is always centered on the $\mathrm{K}$ star, not on the $\mathrm{F}$ star as might be expected from an accretion disk.

\section{SUMMARY AND DISCUSSION}

Our new data for SZ Psc give further clues about the distribution of spots and active regions in chromospherically active stars, although they clearly do not define these distributions unambiguously. We have compared a light curve for 1978, when SZ Psc was relatively unspotted, with one for 19931994, when spots were more prevalent. The difference between these two curves shows that the light was significantly depressed by spots at all orbital phases in 1993-1994. Fitting this depression with a spot model shows that spots were distributed all around the cooler component of SZ Psc. The star was much more heavily spotted than it had been in 1978, even though there must have been a few spots detected in 1978.

These spots depressing the light in 1993-1994 must have been relatively small, both to fit the light curve and to give acceptably small line profile distortions. Two- or three-spot solutions cannot account for the light loss, because so few spots would necessarily have been big enough to distort contemporaneous optical line profiles unacceptably. In fact, our preferred distribution of eight spots for the light-curve solution contained three to four spots that were so large that they were inconsistent with the Doppler profiles. These profile distortions imply $\gtrsim 15$ spots $\left(r \lesssim 8^{\circ}-10^{\circ}\right)$ are needed to fit the light-curve depression. In further analysis with spots preferentially at high latitude, we again found that the large spots must break up into much smaller ones to fit the line profiles. We have belabored the question of continental spots because they seems to be recurring in analyses of long-term photometry, such as Jarvinen et al. (2005a, 2005b). These analyses are a reprise of the Hall two-spot model (Hall et al. 1990) with more sophisticated mathematics. To wit, the Figure 3's of Jarvinen et al. are completely equivalent to the undifferentiated migration diagrams of early two-spot analyses (e.g., Fried et al. 1983, Fig. 2; Strassmeier 
et al. 1988, Fig. 4), with the spots forced onto two continuous parallel sequences covering many years, which were inconsistent with the variation of spot strength with time (e.g., Hall et al. 1990; Henry et al. 1995).

Active regions are numerous and spread around the star. There are probably $\gtrsim 20$ of them, possibly several hundred. These active regions may have been saturating the photosphere between the spots, and not simply confined to the fringes of spots with luminosity proportional to spot area. However, the data are too noisy to prove this, and the analysis too model dependent. We failed to detect eclipses of active regions, although we extensively observed two secondary eclipses with IUE. This null result means merely that the $\sim 4 \%$ of the cool star's area covered by the hot star did not contain a disproportionately large fraction of the chromosphere and transition region.

We have detected a flare in one observation of transition region emission. With the similar flare observed by Doyle et al. (1994a), we have now recorded two flares in 26 shortwavelength IUE spectra of SZ Psc. This new flare may have been relatively short-lived, since it was not clearly detected in either of the flanking Mg II observations. However, we have only one SWP spectrum in which the flux was significantly greater than average, and hence no way of knowing the C IV flux in the hours preceding our flare spectrum.

Our new light-curve solution, which is constrained to fit the broad Doppler profiles of the K star, finds the K star uncomfortably close to its Roche lobe. Since the K star is more massive than its hotter companion, the first contact with the Roche lobe would seem to be sufficient to initiate the rapid mass exchange thought to transform binaries like SZ Psc into Algol systems. A further mystery of this system is the slow rotation of the F star, which would be rotating several times as fast if it were synchronous with the orbit. RS CVn itself shares this property of slow rotation (see the observed and calculated profiles of the F star in Figs. 5 and 8 of Eaton et al. 1993). These measured rotations may simply represent what is expected for an aging main-sequence $\mathrm{F}$ star, but they certainly mean that neither system has undergone mass exchange or that either $\mathrm{F}$ star has gained appreciable rotational angular momentum from its K companion's wind.

\subsection{The Organization of Spots}

Although in 1996 we proposed explaining the temporal variations of chromospherically active stars with a random-spots model, which could explain the rotational modulation, shortterm fluctuations, and putative "cycles" of these stars, there have been precious few studies of either "cyclic" behavior or Doppler images that have addressed the applicability of such a model. A notable exception is a series of papers by S. V. Jeffers (Jeffers 2005; Jeffers et al. 2005, 2006a, 2006b) that used very precise spectrophotometry of SV Cam from the Hubble Space Telescope (HST) to explore the reality of the existence of large numbers of small spots on a chromospherically active star and to test the notion of large polar spots. The main conclusion of that analysis is that the supposedly unspotted photosphere contains large numbers of microscopic spots that lower the surface brightness without giving any eclipse effects. Another is that SV Cam has rather large polar caps (spots) in spite of there being few of the moderate-sized spots of the sort detected in Doppler images. The spot distribution Jeffers derived is probably inconsistent with our random-spots model. The many microscopic spots would not give the variations expected from statistical fluctuations, and the existence of polar caps is completely outside the model. The analysis of SV Cam is somewhat weak, however, in that the existence of those microscopic spots depends critically on a parallax known to $10 \%$, and hence to absolute fluxes known at best to $20 \%$, while $1 \sigma$ is not an absolute limit. The polar caps might be better constrained, but they would seem to be less important (through the factor $\left[r_{\mathrm{sec}} / d_{\mathrm{Hipp}}\right]^{2} F_{\mathrm{sec}}$ in eq. [8] of Jeffers et al. [2005]) if we adjust the parallax to eliminate the microscopic spots. The existence of large polar spots, a common feature of Doppler images, has never been tested sufficiently, in our opinion (but see remarks by N. Piskunov at the end of Strassmeier [2001]). In fact, such testing seemed to be effectively ended by the untimely death of Brendan Byrne. This work on SV Cam represents an exciting new direction in the attempt to test ideas about spot distributions. Although having the broad wavelength coverage of $H S T$ was nice, Jeffers et al. could have done most of their work from the ground. This means one could easily monitor SV Cam over the years, looking for photometric and profile changes from radical changes in the spot distribution.

SZ Psc has some distinct advantages over SV Cam as a vehicle for using eclipsing binaries to assess the locations of spots and the existence of polar spots: it is brighter, it has a much broader Doppler profile, and it has an F component that could serve much more reliably as a comparison star for the spotted component than would the cooler component of SV Cam, itself likely spotted and variable. However, SZ Psc is a more difficult system to observe effectively because of its integral-day period. Yet with good phase coverage, it would give us several tests of ideas about spots. For instance, the broad Doppler profile gives many resolution elements on the disk for any spectrograph. Furthermore, precise photometry of the secondary eclipse might detect eclipses of some of the noncircumpolar spots that we have shown must exist, and thus restrict their latitudes. Likewise, the $70^{\circ}$ inclination means that Doppler images would sample spots of different latitude differently in the two hemispheres and possibly discriminate between equatorial spots and those at higher latitudes. Finally, the seemingly uncomplicated light curve for 1978 shows that with a great deal of luck, one could eventually catch this star in a relatively unspotted state and find out just what such a state represents.

We would like to thank the staffs of the National Solar Observatory, particularly Paul Avellar and Trudy Tilleman, and 
IUE observatory for help with planning and taking the observations. We are especially thankful to Lyla Taylor for help with handling and analyzing some of the IUE data. We are also grateful to the late Brendan Byrne and to Suzanne Hawley for helping with the line identifications in the flare spectrum. This research has been supported by NASA grants NAG 8-111, NAG 8-1014, NCCW-0085, and NCC5-511, and by NSF grants HRD 9550561 and HRD-9706268.

\section{REFERENCES}

Andrews, A. D., et al. 1988, A\&A, 204, 177

Baliunas, S. L., Avrett, E. H., Hartmann, L., \& Dupree, A. K. 1979, ApJ, 233, L129

Baliunas, S. L., \& Dupree, A. K. 1982, ApJ, 252, 668

Bopp, B. W. 1981, AJ, 86, 771

Bopp, B. W., \& Talcott, J. C. 1978, AJ, 83, 1517 1980, AJ, 85, 55

Buzasi, D. L., Ramsey, L. W., \& Huenemoerder, D. P. 1987, ApJ, 322, 353

Cram, L. E., \& Mullan, D. J. 1979, ApJ, 234, 579

Cuny, Y. 1965, in Proc. Second Harvard-Smithsonian Conference on Stellar Atmospheres, the Formation of Spectrum Lines, ed. E. H. Avrett, O. J. Gingerich, \& C. A. Whitney (SAO Spec. Rept. No. 167; Cambridge: SAO), 275

Danezis, E., Antonopoulou, E., Theodossiou, E., \& Mathioudakis, M. 1992, Ap\&SS, 187, 307

Doyle, J. G., Byrne, P. B., \& van den Oord, G. H. J. 1989, A\&A, 224,153

Doyle, J. G., Mitrou, C. K., Mathioudakis, M., \& Antonopoulou, E. 1994a, A\&A, 283, 522

Doyle, J. G., Mitrou, C. K., Mathioudakis, M., Avgoloupis, S., Mavridis, L. N., Varvoglis, P. P., Graos, F., \& Antonopoulou, E. 1994b, A\&A, 291, 135

Eaton, J. A. 1995, AJ, 109, 1797

Eaton, J. A., \& Hall, D. S. 1979, ApJ, 227, 907

Eaton, J. A., Henry, G. W., Bell, C., \& Okorogu, A. 1993, AJ, 106, 1181

Eaton, J. A., Henry, G. W., \& Fekel, F. C. 1996, ApJ, 462, 888

Eaton, J. A., Scaltriti, F., Cerruti-Sola, M., Sarma, M. B. K., Ausekar, B. D., Catalano, S., \& Rodonò, M. 1982, Ap\&SS, 82, 289

Eaton, J. A., \& Williamson, M. H. 2004, Proc. SPIE, 5496, 710

Fried, R. E., et al. 1983, Ap\&SS, 93, 303

Genet, R. M., Boyd, L. J., Kissell, K. E., Crawford, D. L., Hall, D. S., Hayes, D. S., \& Baliunas, S. L. 1987, PASP, 99, 660

Hall, D. S. 1976, in IAU Colloq. 29, Multiple Periodic Variable Stars, Part I, ed. W. S. Fitch (Dordrecht: Reidel), 287

1990, in Active Close Binaries, ed. C. Ibanoglu (Dordrecht: Kluwer), 95

Hall, D. S., Henry, G. W., \& Sowell, J. R. 1990, AJ, 99, 396

Hall, D. S., \& Kreiner, J. M. 1980, Acta Astron., 30, 387

Hatzes, A. 1995, AJ, 109, 350

Henry, G. W. 1995, in ASP Conf. Ser. 79, Robotic Telescopes: Current Capabilities, Present Developments, and Future Prospects for Automated Astronomy, ed. G. W. Henry \& J. A. Eaton (San Francisco: ASP), 44

Henry, G. W., Eaton, J. A., Hamer, J., \& Hall, D. S. 1995, ApJS, 97, 513

Huenemoerder, D. P., \& Ramsey, L. W. 1984, AJ, 89, 549

Jakate, S. M., Bakos, G. A., Fernie, J. D., \& Heard, J. F. 1976, AJ, 81,250

Jarvinen, S. P., Berdyugina, S. V., \& Strassmeier, K. G. 2005a, A\&A, 440,735

Jarvinen, S. P., Berdyugina, S. V., Touminen, I., Cutispoto, G., \& Bos, M. 2005b, A\&A, 432, 657

Jefferies, J. T., \& Thomas, R. N. 1959, ApJ, 129, 401

Jeffers, S. V. 2005, MNRAS, 359, 729
Jeffers, S. V., Aufdenberg, J. P., Hussain, G. A. J., Collier Cameron, A., \& Holzwarth, V. R. 2006a, MNRAS, 367, 1308

Jeffers, S. V., Barnes, J. R., Collier Cameron, A., \& Donati, J.-F. 2006b, MNRAS, 366, 667

Jeffers, S. V., Collier Cameron, A., Barnes, J. R., Aufdenberg, J. P., \& Hussain, G. A. J. 2005, ApJ, 621, 425

Johnson, H. R., \& Klinglesmith, D. A. 1965, in Proc. Second HarvardSmithsonian Conference on Stellar Atmospheres, the Formation of Spectrum Lines, ed. E. H. Avrett, O. J. Gingerich, \& C. A. Whitney (SAO Spec. Rept. No. 167; Cambridge: SAO), 221

Kalimeris, A., Mitrou, C. K., Doyle, J. G., Antonopoulou, E., \& Rovithis-Livaniou, H. 1995, A\&A, 293, 371

Kang, Y. W., Lee, W.-B., Kim, H.-I., \& Oh, K.-D. 2003, MNRAS, 344,1227

Kreiner, J. M., Kim, C.-H., \& Nha, I.-S. 2000, An Atlas of $O-C$ Diagrams of Eclipsing Binary Stars (Krakow: Wydawnctwo Naukowe Ap)

Kürster, M., Schmitt, J. H. M. M., \& Cuitispoto, G. 1994, A\&A, 289, 899

Lanza, A. F., Rodonò, M., Mazzola, L., \& Messina, S. 2001, A\&A, 376,1011

Noah, P. V., Bopp, B. W., \& Fekel, F., Jr. 1987, in Fifth Cambridge Workshop on Cool Stars, Stellar Systems, and the Sun, ed. J. L. Linsky \& R. E. Stencel (Berlin: Springer), 506

Olah, K., Kovari, Zs., Bartus, J., Strassmeier, K. G., Hall, D. S., \& Henry, G. W. 1997, A\&A, 321, 811

Olah, K., \& Strassmeier, K. G. 2002, Astron. Nachr., 323, 361

Popper, D. M. 1988, AJ, 96, 1040

Rodonò, M., Lanza, A. F., \& Catalano, S. 1995, A\&A, 301, 75

Scarfe, C. D., Batten, A. H., \& Fletcher, J. M. 1990, Publ. Dominion Astrophys. Obs. Victoria, 18, 21

Schmitt, J. H. M. M., \& Stern, R. A. 1994, in ASP Conf. Ser. 64, Eighth Cambridge Workshop on Cool Stars, Stellar Systems, and the Sun, ed. J.-P. Calliault (San Francisco: ASP), 134

Sciortino, S., Micela, G., Khashyap, V., Harnden, F. R., Jr., \& Rosner, R. 1994, in ASP Conf. Ser. 64, Eighth Cambridge Workshop on Cool Stars, Stellar Systems, and the Sun, ed. J.-P. Calliault (San Francisco: ASP), 140

Strassmeier, K. G. 2001, in ASP Conf. Ser. 223, Eleventh Cambridge Workshop on Cool Stars, Stellar Systems, and the Sun, ed. R. J. Garcia Lopez, R. Rebolo, \& M. R. Zapatero Osorio (San Francisco: ASP), 271

. 2004, in ASP Conf. Ser. 318, Spectroscopically and Spatially Resolving the Components of the Close Binary Stars, ed. R. W. Hidlitch, H. Hensberge, \& K. Pavlovski (San Francisco: ASP), 69 Strassmeier, K. G., Hall, D. S., Fekel, F. C., \& Scheck, M. 1993a, A\&AS, 100, 173

Strassmeier, K. G., et al. 1988, A\&A, 192, 135 1993b, A\&A, 268, 671

Tunca, Z. 1984, Ap\&SS, 105, 23

Weiler, E. J. 1978a, MNRAS, 182, 77 . 1978b, AJ, 83, 795

Van Hamme, W., \& Wilson, R. E. 1993, MNRAS, 262, 220

Zeilik, M. 1992, in ASP Conf. Ser. 26, Seventh Cambridge Workshop on Cool Stars, Stellar Systems, and the Sun, ed. M. S. Giampapa \& J. A. Bookbinder, (San Francisco: ASP), 346 